\title{
Nolz1 promotes striatal neurogenesis through the regulation of retinoic acid signaling
}

\author{
Noelia Urbán 1,2,3, Raquel Martín-Ibáñez ${ }^{1,2,4}$, Cristina Herranz ${ }^{1,2,4}$, Miriam Esgleas ${ }^{1,2,4}$, Empar Crespo 1,2,4, \\ Monica Pardo ${ }^{1}$, Ivan Crespo-Enríquez ${ }^{5}$, Héctor R Méndez-Gómez ${ }^{2,6}$, Ronald Waclaw ${ }^{7}$, Christina Chatzi ${ }^{8}$, \\ Susana Álvarez ${ }^{9}$, Rosana Álvarez ${ }^{9}$, Gregg Duester ${ }^{8}$, Kenneth Campbell ${ }^{7}$, Angel R de Lera ${ }^{9}$, Carlos Vicario-Abejón ${ }^{2,6}$, \\ Salvador Martinez ${ }^{5}$, Jordi Alberch ${ }^{1,2,4}$, Josep M Canals 1,2, $^{*}$
}

\begin{abstract}
Background: Nolz1 is a zinc finger transcription factor whose expression is enriched in the lateral ganglionic eminence (LGE), although its function is still unknown.

Results: Here we analyze the role of Nolz1 during LGE development. We show that Nolz1 expression is high in proliferating neural progenitor cells (NPCs) of the LGE subventricular zone. In addition, low levels of Nolzl are detected in the mantle zone, as well as in the adult striatum. Similarly, Nolz1 is highly expressed in proliferating LGE-derived NPC cultures, but its levels rapidly decrease upon cell differentiation, pointing to a role of Nolz1 in the control of NPC proliferation and/or differentiation. In agreement with this hypothesis, we find that Nolz1 overexpression promotes cell cycle exit of NPCs in neurosphere cultures and negatively regulates proliferation in telencephalic organotypic cultures. Within LGE primary cultures, Nolzl over-expression promotes the acquisition of a neuronal phenotype, since it increases the number of $\beta$-III tubulin (Tuj1)- and microtubule-associated protein (MAP)2-positive neurons, and inhibits astrocyte generation and/or differentiation. Retinoic acid (RA) is one of the most important morphogens involved in striatal neurogenesis, and regulates Nolz1 expression in different systems. Here we show that Nolz1 also responds to this morphogen in E12.5 LGE-derived cell cultures. However, Nolz1 expression is not regulated by RA in E14.5 LGE-derived cell cultures, nor is it affected during LGE development in mouse models that present decreased RA levels. Interestingly, we find that Gsx2, which is necessary for normal RA signaling during LGE development, is also required for Nolz1 expression, which is lost in Gsx2 knockout mice. These findings suggest that Nolz1 might act downstream of Gsx2 to regulate RA-induced neurogenesis. Keeping with this hypothesis, we show that Nolz1 induces the selective expression of the RA receptor (RAR) $\beta$ without altering RAR $\alpha$ or RAR $\gamma$. In addition, Nozl1 over-expression increases RA signaling since it stimulates the RA response element. This RA signaling is essential for Nolz1-induced neurogenesis, which is impaired in a RA-free environment or in the presence of a RAR inverse agonist. It has been proposed that Drosophila Gsx2 and Nolz1 homologues could cooperate with the transcriptional co-repressors Groucho-TLE to regulate cell proliferation. In agreement with this view, we show that Nolz1 could act in collaboration with TLE-4, as they are expressed at the same time in NPC cultures and during mouse development.
\end{abstract}

Conclusions: Nolz1 promotes RA signaling in the LGE, contributing to the striatal neurogenesis during development.

\footnotetext{
* Correspondence: jmcanals@ub.edu

'Department of Cell Biology, Immunology and Neuroscience, Faculty of Medicine, IDIBAPS, Universitat de Barcelona, C/Casanova 143, 08036

Barcelona, Spain

Full list of author information is available at the end of the article
}

\section{Ciomed Central}

C 2010 Urbán et al; licensee BioMed Central Ltd. This is an Open Access article distributed under the terms of the Creative Commons Attribution License (http://creativecommons.org/licenses/by/2.0), which permits unrestricted use, distribution, and reproduction in any medium, provided the original work is properly cited. 


\section{Background}

During the first stages of striatal development, neurons arise from the ventricular zone (VZ), which is mainly composed of neuroepithelial cells [1,2]. Around embryonic day (E)11.5, these cells divide asymmetrically; giving rise to radial glial cells and neural progenitor cells (NPCs). Radial glial cells have extensions that contact with the ventricular lumen and with more differentiated inner zones of the developing striatum, but their cell bodies remain in the VZ [3-5]. In contrast, NPCs leave the $\mathrm{VZ}$ to proliferate and generate the so-called subventricular zone (SVZ). Within the dorsal telencephalon, the SVZ is a thin layer of cells, mainly formed of NPCs $[4,6]$. However, in the ventral telencephalon, it becomes a prominent structure that has been shown to be the main source of striatal neurons and glia. Striatal projecting neurons are born in the SVZ of the lateral ganglionic eminence (LGE) whereas the medial ganglionic eminence will give rise to cortical and striatal interneurons.

Retinoic acid (RA) is one of the morphogens that participates in the specification and differentiation of the intermediate position of the LGE in the telencephalon [7]. However, RA sources during telencephalon development are still unclear. Three different retinaldehyde dehydrogenases (Raldhs), the limiting enzymes for RA synthesis, have been described in the central nervous system. Two of them, Raldh2 and Raldh3, are expressed in the otic vesicles and frontonasal ectoderm, respectively, as early as E8.5 [8-10]. Thus, it is unlikely that RA from these sources could reach the intermediate telencephalon. Around E12.5, Raldh3 expression appears in the LGE, providing the first known source of RA in the striatum $[9,11]$. During this period, the expression of the RA receptors (RARs) RAR $\alpha$ and RAR $\beta$ is high in the ventral telencephalon and it has been shown that RAR $\beta$ stimulation mediates gene regulation in the developing telencephalon, particularly on striatal neuronal populations [12] where $95 \%$ of neurons are GABAergic [13]. In addition, RA increases the number of GABAergic neurons in differentiating mouse embryonic stem cells through the regulation of RAR $\beta[14,15]$. Some transcription factors are of great importance for RA signaling in the LGE. Among these is the homeobox transcription factor Gsx2, which is essential for correct striatal development $[16,17]$ and for Raldh3 expression. Raldh3 levels are severely reduced or lost in mice deficient for Gsx2 or Gsx1 and Gsx2 [18].

To exert its effect during development, RA binds specific RARs and, thereafter, regulates the expression of some transcription factors that contain a RA response element (RARE) in their promoter. One of these transcription factors is Nolz1, which is induced by RA in the PC12 neural cell line and during developing chick spinal cord $[19,20]$. Nolz1 is a member of the NocAElbow (elB)-Tlp (NET) family of transcription factors, which are involved in patterning and differentiation during development in all studied species [21-24]. In zebrafish, two members of the NET family have been identified, $n l z 1$ and $n l z 2$, and both have been described to be essential for boundary formation in the rombencephalon [23,24]. Nlz2 is also expressed in more anterior structures, including the telencephalic vesicles, where it is supposed to play similar roles in regionalization [25]. A Nlz2 homolog in rodents was called Nolz1 (also known as $Z f p 503$ ), which is expressed during nervous system development in several regions, including the hypothalamus and spinal cord. However, its highest expression is localized in the ventral LGE (vLGE) $[19,26]$. Of note, Nolz1 is totally absent in close telencephalic structures such as the medial ganglionic eminence and the pallidum, including the dorsal LGE (dLGE) [19], suggesting a highly specific striatal function. However, this family of transcription factors cannot directly interact with DNA, indicating that they need to interact with other transcription factors. In Drosophila, it has been shown that elbow B (elB) interacts with Groucho (Gro) proteins, forming large complexes of proteins that act as transcriptional co-repressors [27].

Despite its characterized pattern of expression, the function of Nolz1 during telencephalic development has not been analyzed yet. Here we studied the role of Nolz1 during vLGE development and the relationship between Nolz1 and the RA pathway within the LGE. Our results show that Nolz1 induces cell cycle exit and promotes neuronal differentiation. In addition, we also demonstrate that Nolz1 expression is temporarily regulated by RA during early striatal development. Finally, we show that Nolz1 contributes to neuronal differentiation through the increase of RA signaling.

\section{Results}

Nolz1 expression is regulated during striatal development In order to analyze whether the temporal expression pattern of Nolz1 protein resembles that of Nolz1 mRNA, a polyclonal antibody was raised against 12 amino acids of the amino terminus of the Nolz1 protein sequence (Figure 1A). Western blotting of NPC lysates showed that the antibody recognizes a unique specific band when compared to the pre-serum blotted membrane (Figure 1B). In addition, dot blot analyses demonstrated the specificity of the serum (Figure 1C), which could be blocked by the competitive incubation of the antibody with the Nolz1 peptide (Figure 1D). Using this Nolz1 polyclonal antibody, we performed western blot analysis in striatal samples from E14.5, E18.5, postnatal 


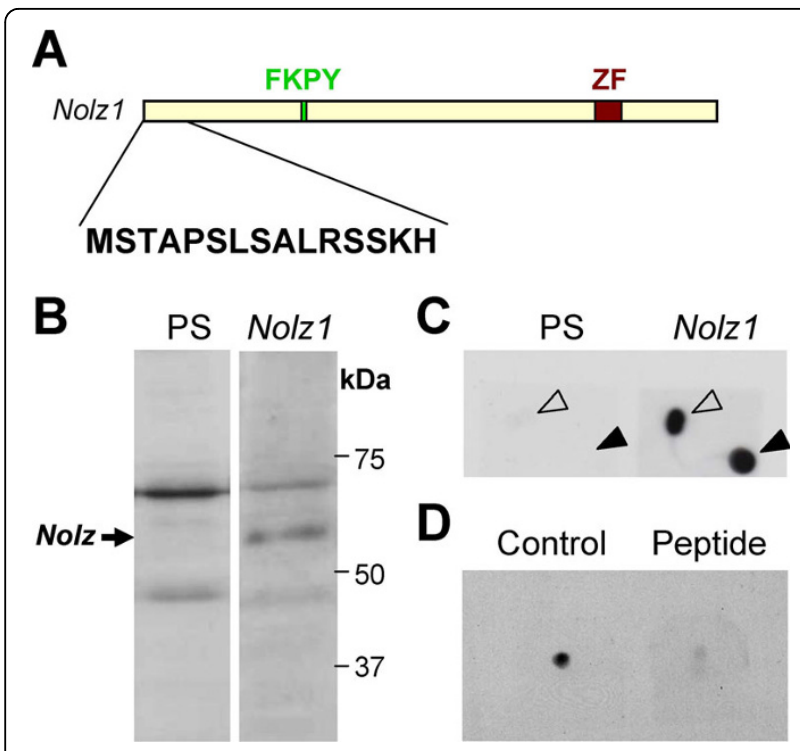

Figure 1 Characterization of the anti-Nolz1 polyclonal antibody. (A) Representation of Nolz1 showing the peptide against which the polyclonal antibody was raised. FKPY, Groucho consensus binding site; ZF, zinc-finger domain. (B) Western blots of NPC protein extracts, showing endogenous Nolz1 expression. Membranes were incubated with a rabbit serum obtained before (pre-serum; PS) or after (Nolz1) immunization. The specific band corresponding to Nolz1 (63 kDa; arrow) is visible only with the antiNolz1 antibody. (C) Dot-blot against Nolz1 pure peptide (upper drop; open arrowheads) or Keyhole limpet hemocyanin (KLH)conjugated Nolz1 peptide (lower drop; closed arrowheads) incubated with rabbit serum before (pre-serum; PS) and after (Nolz1) immunization, showing Nolz1 antibody specificity. (D) Nolz1 pure peptide dots immuno-blotted with the anti-Nolz1 polyclonal antibody pre-incubated in the presence (Peptide) or in the absence (Control) of Nolzl peptide.

day (P)3 and adult mice. Our results showed that Nolz1 levels are high at embryonic stages, being downregulated postnatally (Figure $2 \mathrm{~A}$ ). Within the adult, low levels of Nolz1 protein remained in the striatum while no expression was detected in the subependymal zone (Figure 2A). In situ hybridization for Nolz1 showed high levels of mRNA in the SVZ of the vLGE at E14.5 (Figure 2B). No signal was detected in the dLGE (Figure 2B), which gives rise to olfactory bulb interneurons during development [28]. Low levels of Nolz1 mRNA were also detected in the mantle zone (MZ; Figure 2B,D), where it partially co-localized with Tuj1-positive neurons (Figure $2 \mathrm{D}, \mathrm{E})$. In the adult brain, similar to the protein results, Nolz1 mRNA was not detected in the subependymal zone, although faint expression could still be detected in the striatum by in situ hybridization (Figure 2C).

\section{Nolz1 regulates NPC homeostasis in the LGE}

The high levels of Nolz1 expression in the SVZ of the vLGE suggest it has a role in the regulation of NPC homeostasis. Thus, to analyze the function of Nolz1 in NPCs, we generated neurospheres from the LGE of E14.5 mice. Nolz1 expression was analyzed in proliferating cells and at 3 and 6 days in vitro (DIV) after the induction of neurosphere differentiation (Figure 3A,B). We observed that Nolz1 expression levels were high in non-differentiated cells, while its expression decreased during the differentiation process for both mRNA (Figure 3A) and protein (Figure 3B). These results were coincident with the expression pattern of Nolz1 in vivo, which mainly corresponded to the NPC-containing SVZ (Figure 2B).

We next analyzed whether Nolz1 regulates NPC proliferation. Over-expression of human Nolz (hNolz) in NPCs led to high $h N o l z$ mRNA levels but a significant decrease in endogenous mouse Nolz1 expression (Figure 4A), suggesting tight regulation of mouse Nolz1 levels. When we analyzed Nolz protein levels with a Nolz1 antibody that detects mouse and human isoforms, we found a net increase in Nolz protein levels with respect to control transfected cells (Figure 4B). hNolz-over-expressing neurospheres showed a $19.5 \%$ reduction in the number of proliferating bromodeoxyuridine (BrdU)-positive NPCs with respect to control transfected cells (Figure 4D,E). Double immunocytochemistry showed that most, but not all, of the $h N o l z$-over-expressing cells were negative for BrdU (Figure 4D). To verify that the reduction in BrdUpositive cells was due to cell cycle exit and not to variations in its duration, we measured the cell cycle time of transfected NPCs as described elsewhere [29] and did not find any difference between control and hNolz-overexpressing neurospheres (DsRED, $25.29 \mathrm{~h}$; hNolz-DsRED, $24.88 \mathrm{~h}$ ). In addition, we analyzed the index of cell cycle exit as described previously by Chenn and Walsh [30]. This demonstrated that $h N o l z$ over-expression produces a significant increase of $162 \%$ in the cell cycle exit index (Figure 4G). Since the reduction in the number of BrdUpositive NPCs may also be due to cell death, we also counted the number of apoptotic nuclei of $h N o l z$-overexpressing NPCs with respect to control enhanced green fluorescent protein (EGFP)-transfected NPCs. No cell death was observed in any condition (data not shown).

In addition, the effect of Nolz1 silencing was also analyzed by using a cocktail of three different Nolz1 small interfering RNAs (siRNAs) at a concentration of $2 \mu \mathrm{M}$, which led to $60 \%$ inhibition of Nolz1 mRNA (data not shown) and $82 \%$ inhibition of Nolz1 protein expression 2 DIV after transfection (Figure 4C). As expected, transfection of Nolz1 siRNA increased the number of BrdUpositive NPCs (by 18.5\%) in the neurosphere cultures with respect to scrambled negative siRNA control transfection (Figure 4E). Taken together, these findings demonstrate that deregulation of Nolz1 altered the proliferating capacity of NPCs, suggesting that Nolz1 


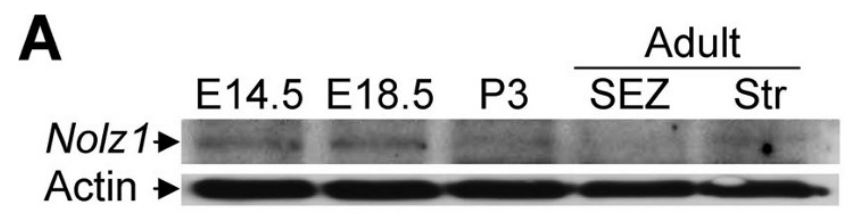

B
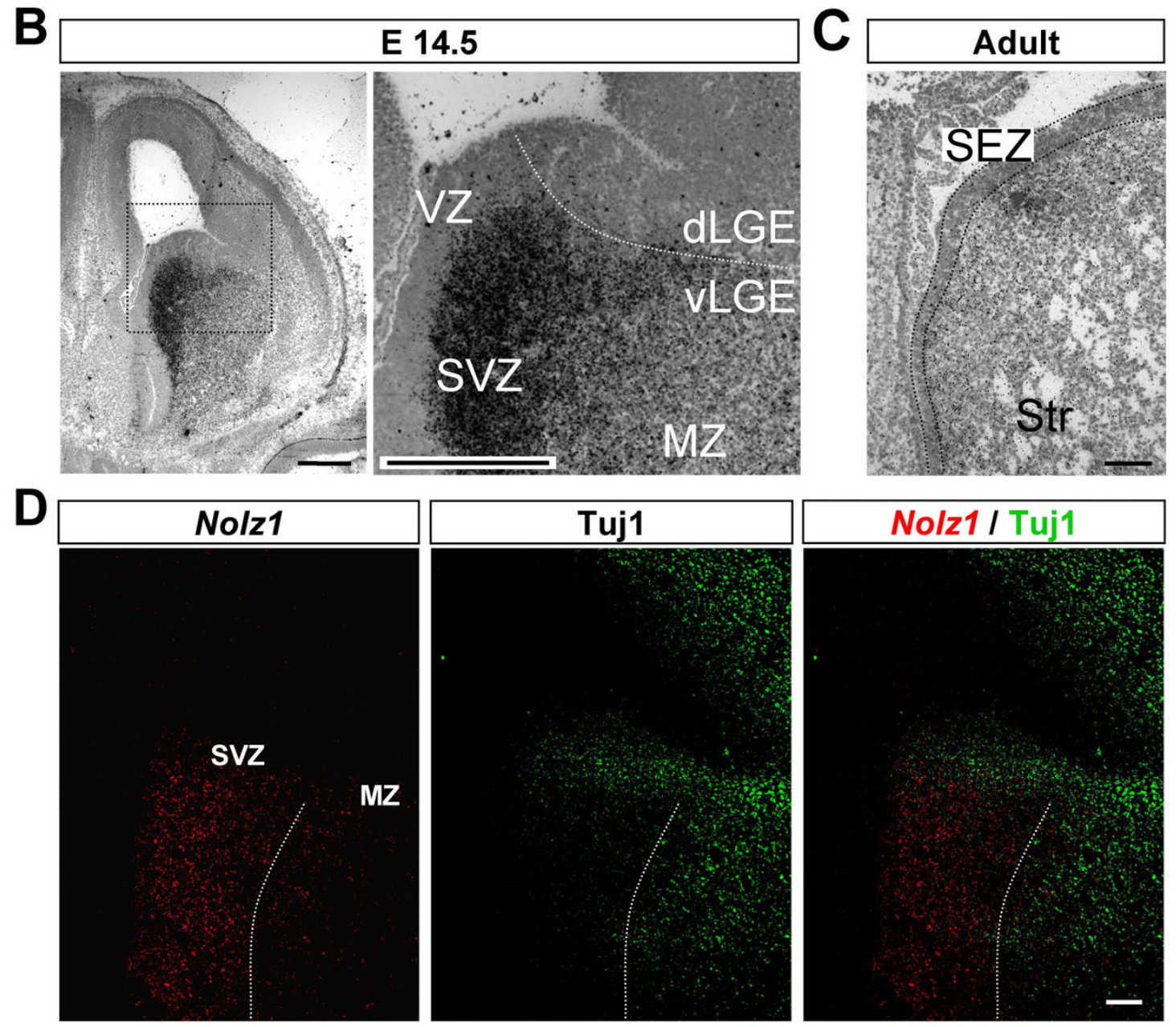

E
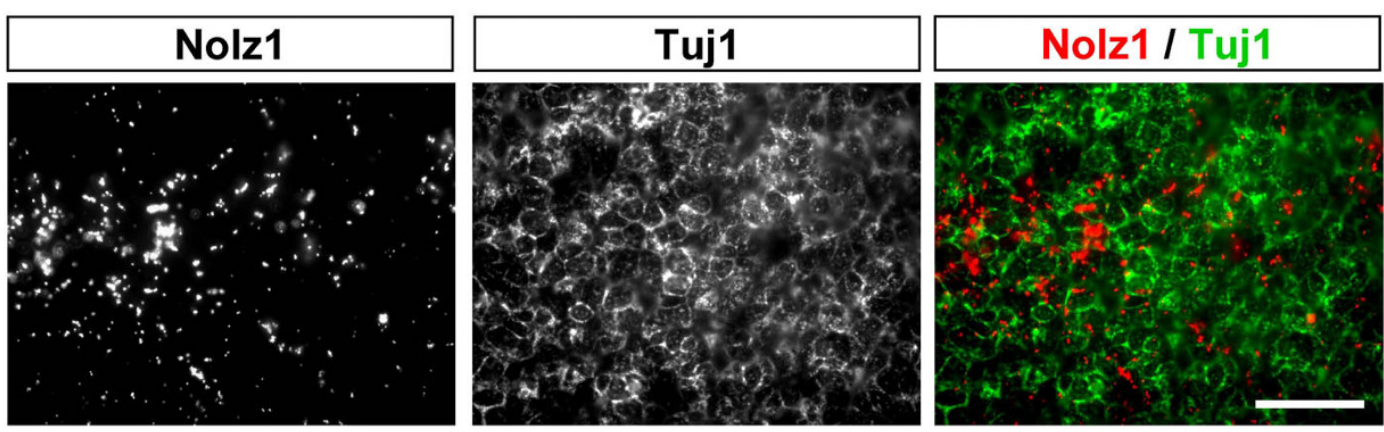

Figure 2 The pattern of Nolz1 protein expression resembles that of Nolz1 mRNA in the LGE. (A) Western blot analysis of striatal samples from different developmental stages showing high levels of Nolz1 protein at embryonic stages, which decrease during postnatal development. Within adult mice, Nolzl expression remains at low levels in the striatum (Str) but is not detectable in the subependymal zone (SEZ). (B) In situ hybridization shows high levels of Nolz1 mRNA in the SVZ of the E14.5 VLGE. Note that the levels of Nolz1 mRNA decrease in the mantle zone (MZ). Scale bars: $600 \mu \mathrm{m}$. (C) Low levels of Nolz1 mRNA are also detected in the adult striatum but not in the subependymal zone. Scale bar: $150 \mu \mathrm{m}$. (D) Double in situ immunohistochemistry shows that within the MZ, Nolz1 expression is located in $\beta$-III tubulin (Tuj-1)-positive cells. Scale bar: $150 \mu \mathrm{m}$. (E) High magnification of (D). Scale bar: $60 \mu \mathrm{m}$. 


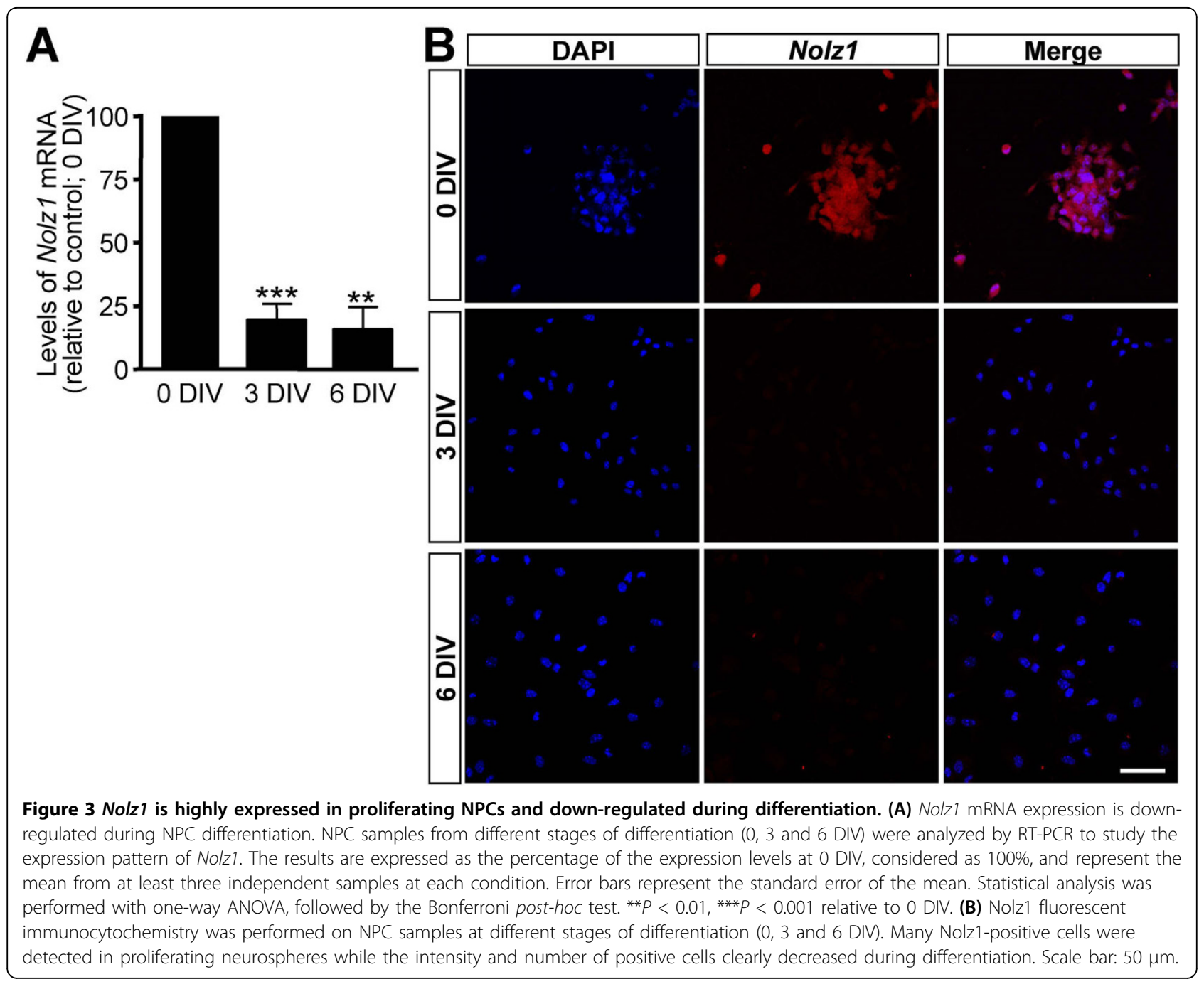

promotes cell cycle exit. Next, we studied the capacity of NPCs to form new neurospheres 5 DIV after $h N o l z$ over-expression or transfection of Nolz1 siRNA (Figure 4F). hNolz negatively regulated NPC self-renewal, as shown by the decrease in the number of new neurospheres formed after hNolz over-expression and the corresponding increase observed after Nolz1 siRNA transfection (Figure 4E, P0). When cells were dissociated and plated again (P1), no differences were observed in any condition studied (Figure $4 \mathrm{E}$ ). These findings indicate a transient effect of Nolz1 and that variation of the levels of this transcription factor does not permanently affect NPC populations since $h N o l z$ over-expression or Nolz1 siRNA was lost after the first passage.

To further confirm the role of Nolz1 in the regulation of NPC proliferation, we next electroporated $h N o l z$ into LGE-derived organotypic cultures from E15.5 embryos and analyzed the number of Ki67-positive cells (Figure 5). Two days after electroporation, $h N o l z$ over-expression reduced the proliferation of NPCs at the SVZ as indicated by the dramatic decrease in the number of Ki67-positive cells with respect to the control electroporated side (Figure 5B).

\section{Nolz1 over-expression promotes the acquisition of a neuronal phenotype in LGE primary cultures}

Reduced proliferation of NPCs could be indicative of neural differentiation. To test this possibility, we first analyzed whether Nolz1 was expressed in differentiated post-mitotic cells in primary cultures derived from E14.5 LGEs (Figure 6A,B). Double immunocytochemistry against Nolz1 and the neural markers Tuj1 or glial fibrillary acidic protein (GFAP) showed that most of the Nolz1-positive cells were positive for the neuronal marker Tuj1, while we could not find any overlap between Nolz1 and the astroglial marker GFAP (Figure 6A,B). We also analyzed the role of Nolz1 on neural differentiation by transfecting LGE primary cultures with 

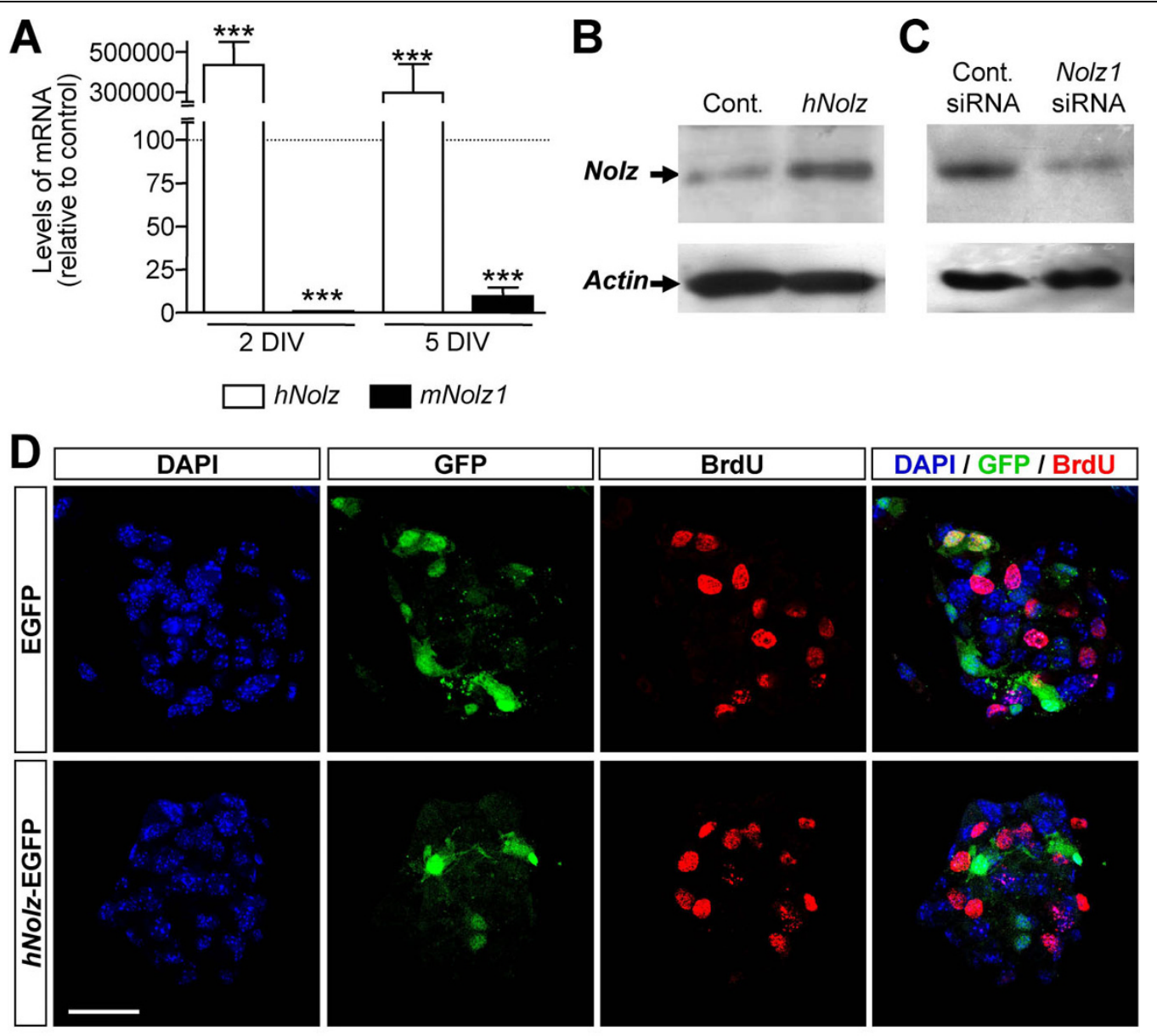

$\mathbf{E}$
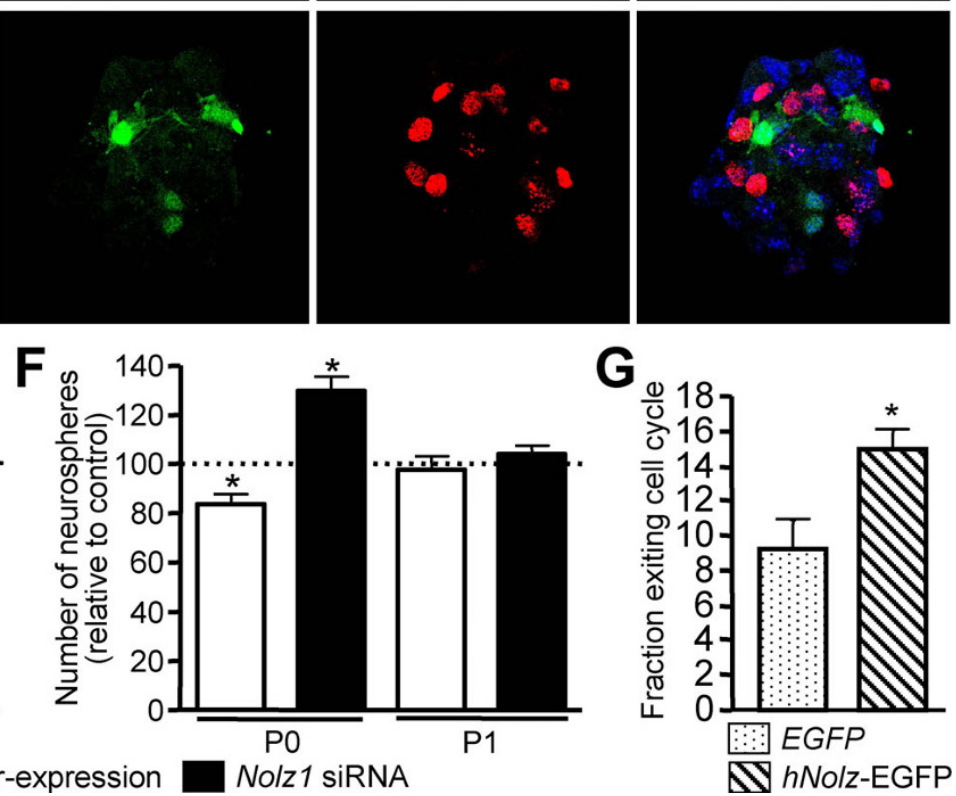

Figure 4 Nolz1 regulates proliferation and self-renewal of NPCs. (A) hNolz over-expression produces a down-regulation of endogenous mouse Nolz1 ( $m$ Nolzl) at 2 and 5 DIV after transfection with hNolzl-DsRED. Values are expressed as the mean percentage of control transfection (DsRED alone), considered as 100\%. (B) Western blot of NPC protein extracts 5 DIV after transfection with DsRED control (Cont.) or hNolz-DsRED (hNolz), showing an increase in Nolz after hNolz transfection. (C) Western blot of NPC protein extracts 2 DIV after transfection of Nolz1 small interfering RNA (siRNA) or control siRNA. The expression of Nolzl decreases about $82 \%$ in the cultures transfected with the Nolzl siRNA relative to those transfected with the control siRNA. (D,E) Over-expression of hNolz in NPCs reduces the number of bromodeoxyuridine (BrdU)-positive cells, whereas Nolz1 siRNA transfection causes an increase in the number of BrdU-positive cells. Results are expressed as the relative number of BrdU-positive cells, standardized to their respective control, considered 100\% (dotted line). (F) Nolz1 over-expression results in a decrease in neurosphere generation, while Nolz1 silencing results in an increase in the number of neurospheres just after transfection (PO, passage 0). When cells are dissociated and plated again (P1, passage 1), no differences were observed in any condition. Results are expressed as the percentage of counted neurospheres with respect to their respective control, considered 100\% (dotted line). (G) Cell-cycle exit index was analyzed as the percentage of BrdU+/Ki67- cells with respect to the total number of BrdU-positive cells after a 3-DIV pulse label. hNolz induces a significant increase in the number of cells that leave the cell-cycle with respect to enhanced green fluorescent protein (EGFP)-over-expressing cells. The results in each graph represent the mean \pm standard error of the mean from at least three independent samples at each condition. Statistical analysis was performed with the Student's $t$-test. ${ }^{*} P<0.05,{ }^{* *} P<0.001$ relative to the respective controls. 

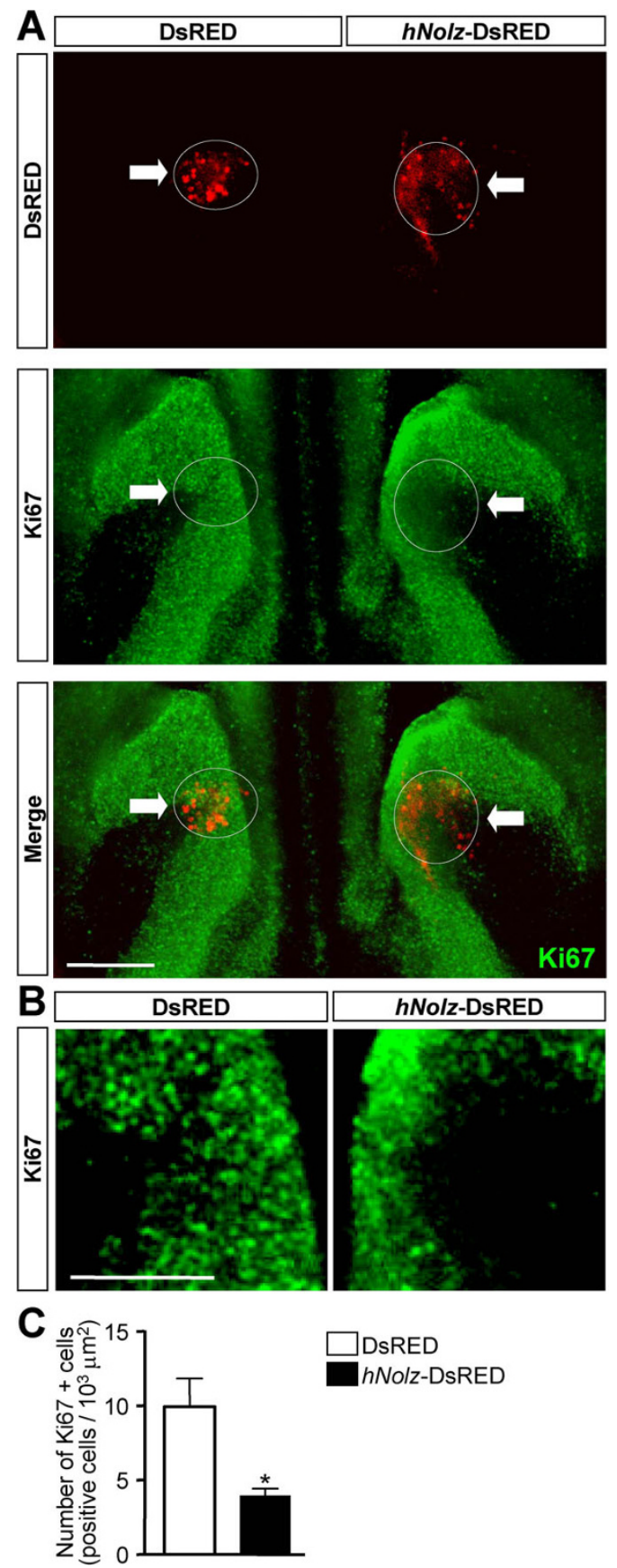

Figure $5 \mathrm{Nolz}$ decreases NPC proliferation in the SVZ of LGE organotypic cultures. (A) Organotypic cultures from E15.5 LGE were electroporated in the VZ/SVZ with $h$ Nolz ( $h$ Nolz-DsRED) or the control vector (DsRED) and stained for Ki67 (white arrows indicate the fluorescent DsRED-expressing cells). Over-expression of hNolz produces a reduction in cell proliferation in the SVZ as shown by the reduction in Ki67-positive cells in the electroporated area. Scale bar: $600 \mu \mathrm{m}$. (B) High magnification of the electroporated area showing the decrease in Ki67-positive cells in the SVZ of the LGE. Scale bar: $300 \mu \mathrm{m}$. (C) Quantification of the number of Ki67-positive cells in the transfected area. The results represent the mean \pm standard error of the mean from four independent experiments. Statistical analysis was performed with the Student's $t$-test; ${ }^{*} P<0.05$. plasmids that express $h N o l z$-EGFP or EGFP alone as control (Figure 6C,D). Five days after transfection, we performed double immunocytochemistry for GFP and the neural precursor gene nestin, the neuronal markers Tuj1 and microtubule-associated protein (MAP) 2 or the astroglial marker GFAP. hNolz-over-expressing cells mainly colocalized with the neuronal markers Tuj 1 and MAP2, although some double GFP-nestin stained cells were also observed (Figure 6C). In contrast, no astroglial cell markers were seen in hNolz transfected cells, as shown by the lack of colocalization between GFP and GFAP (Figure 6C). Interestingly, the quantification of the double positive cells in $h N o l z$ ( $h N o l z$-EGFP) versus control transfected cells (EGFP) demonstrated that $h N o l z$ promotes a neuronal phenotype (Figure 6D). $h N o l z$ over-expression increased the number of both Tuj1- and MAP2-positive neurons, which was accompanied by a reduction in the number of nestin-positive cells (Figure 6D). In addition, no hNolz-over-expressing cells were GFAP-positive astroglia (Figure 6D).

Nolz1 expression is downstream of Gsx2 and its levels are temporarily regulated by RA during striatal development To determine whether RA is needed for Nolz1 expression in striatal cells during development, pregnant mice were fed with a vitamin A (retinol)-deficient diet, which results in a general decrease of RA blood levels [31]. In these animals the levels of $R A R \beta$ were partially reduced (Figure 7A), indicating a decrease in RA levels since it is well known that this receptor is regulated by RA signals. Analysis of Nolz1 expression in E14.5 embryos developed under these vitamin A-deficient levels did not show any difference with respect to expression in regular fed wild-type control embryos (Figure 7B,C). To further confirm that the lack of RA does not affect Nolz1 expression, we analyzed the levels of Nolz1 expression in Raldh3 ${ }^{-1-}$ embryos (Figure 7D), which have completely lost RA activity [11]. Nolz1 expression was not affected by the absence of Raldh 3 in the LGE at E14.5 (Figure 7D), further corroborating the independence of Nolz1 expression from RA signaling in the vLGE at this developmental stage.

To analyze whether Nolz1 expression levels were regulated by RA in vitro, LGE-derived neurospheres were treated with increasing concentrations of RA during 3 DIV and the levels of Nolz1 were analyzed by quantitative PCR (Q-PCR). Nolz1 expression was increased by RA in E12.5-derived NPC cultures (Figure 8A). However, it was not affected in E14.5-derived NPC cultures (Figure 8C). As a well known RA-induced control gene, we analyzed $R A R \beta$, which was clearly upregulated in a dose-dependent manner at both stages (Figure 8B,D). Coincidentally, Nolz1 expression did not change in E14.5 LGE primary cultures treated with RA, although 

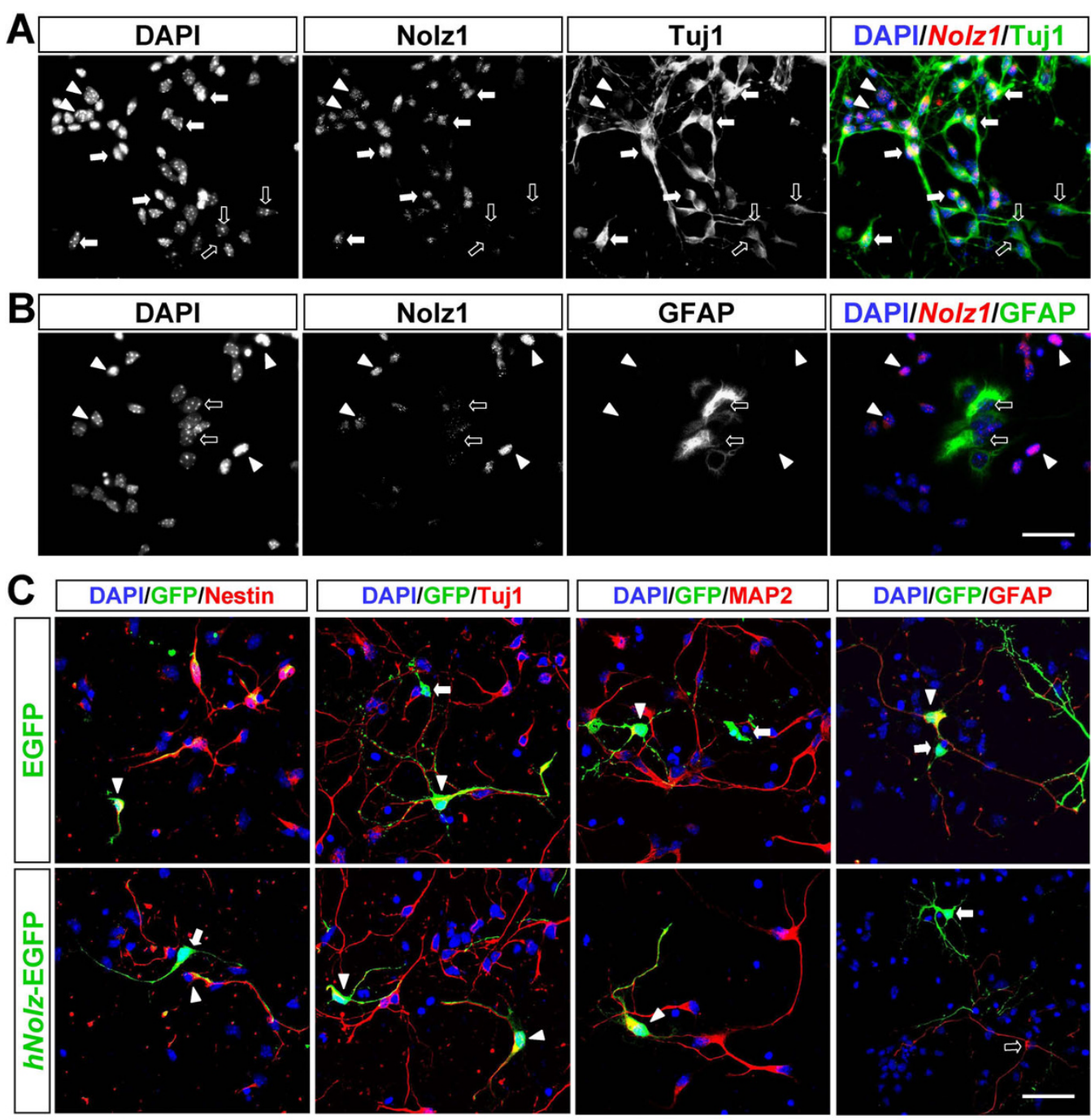

D

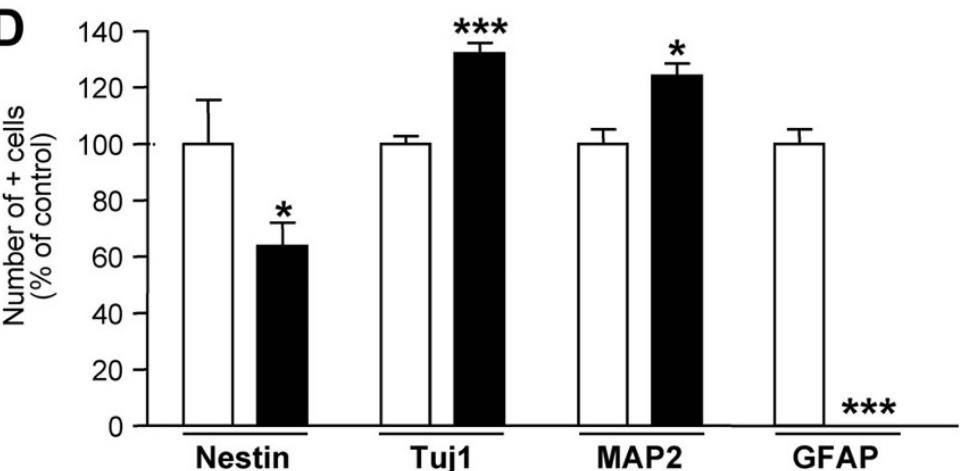

Figure 6 Nolz1 acts as a neurogenic factor in primary LGE cultures. (A,B) Double fluorescent immunocytochemistry performed on primary E14.5 LGE cultures. Nolz1 is expressed in cells positive for the early neuronal marker Tuj1 (A). In contrast, Nolz1 is not detected in cells expressing the astroglial marker glial fibrillary acidic protein (GFAP) (B). White arrows show double positive cells, open arrows show single stained cells for Tuj 1 or GFAP, and white arrowheads show single stained cells for Nolz1. Scale bar: $50 \mu \mathrm{m}$. (C,D) Over-expression of hNolz in LGE primary cultures increased the number of neurons at the expense of glial fates as shown by the increase in the number of Tuj1- or MAP2-positive cells and the complete blockade of astroglial cells. Note that all cells over-expressing hNolz are negative for the GFAP marker (C). (C) White arrows show single stained cells for Nolz1, white arrowheads show double positive cells and open arrows show single GFAP-positive cells. Scale bar: 50 $\mu \mathrm{m}$. (D) The results represent the mean \pm standard error of the mean from at least three independent samples at each condition. Statistical analysis was performed with the Student's $t$-test. ${ }^{*} P<0.05,{ }^{* * *} P<0.001$ relative to EGFP control. 


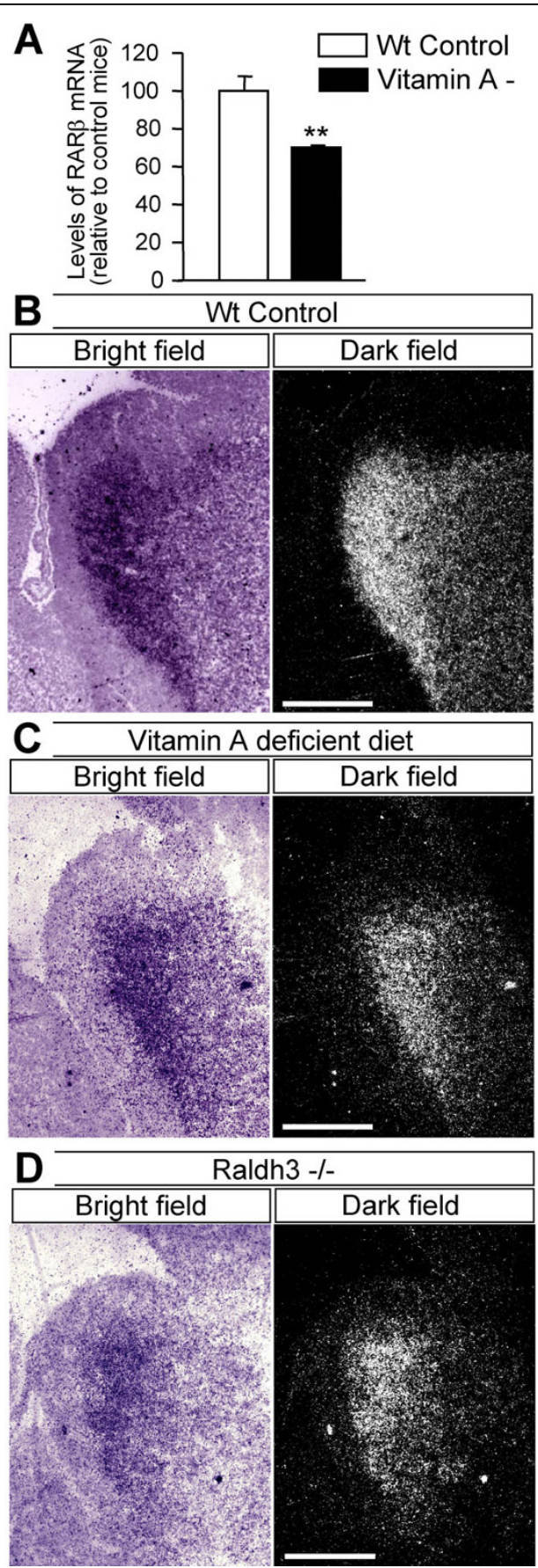

Figure 7 Nolz1 expression does not depend on RA signaling in the LGE in vivo. (A) Embryos developed under a vitamin Adeficient diet (VVitamin A -) had reduced levels of RAR $\beta$ expression compared to wild-type control mice (WT Control). Values are normalized to control (regular diet), considered as $100 \%$, and expressed as the mean of four independent samples for each condition. Error bars represent the standard error of the mean. Statistical analysis was performed with the Student's $t$-test. ${ }^{* *} P<$ 0.01 relative to control. (B) In situ hybridization for Nolzl performed in regular fed wild-type E14.5 embryos. (C) In situ hybridization at E14.5 shows normal Nolz1 expression levels. (D) Similarly, Raldh3 ${ }^{-1}$ embryos at E14.5 show normal Nolz1 expression. Scale bars: $600 \mu \mathrm{m}$.
$R A R \beta$ was also increased in these cultures (Figure $8 \mathrm{E}$ ). Therefore, striatal cultures were competent to increase Nolz1 expression in response to RA treatment at E12.5 but not at later stages. In agreement with these results, when we treated mouse embryonic stem cells with RA, Nolz1 expression was upregulated in a dose-dependent manner (Figure 9), supporting that Nolz1 competence to RA signaling depends on early developmental stages.

Since Gsx2 is essential for the correct expression of several RA-dependent genes, such as Raldh3, in the LGE [18], we also examined the expression of Nolz1 in Gsx2 knockout mice. Nolz1 expression was analyzed by in situ hybridization in knockout mice carrying a single $\left(G s x 2^{+/ E G F P}\right)$ or double substitution of the Gsx2 gene for

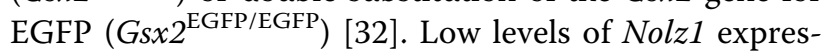
sion remained in the Gsx2 heterozygous mouse vLGE at E14.5 (Figure 10A,B), but no signal was detected in the Gs $x 2^{\text {EGFP/EGFP }}$ mice (Figure 10C), indicating that Gs $x 2$ is critical for Nolz1 expression. On the other hand, overexpression of Gsx2 in NPCs did not lead to an increase in Nolz1 mRNA levels, while it enhanced the levels of Raldh3 mRNA (Figure 10D), which has been shown to be regulated by Gsx2 [18]. These findings indicate that Gsx2 is essential but not sufficient to induce Nolz1 expression.

\section{RA signaling is necessary to induce Nolz1-dependent neurogenesis}

The specific combination of RA receptors defines the competence of cells to respond to RA. Thus, we next analyzed whether Nolz1 expression regulates RARs. We measured $R A R \alpha, R A R \beta$ and $R A R \gamma$ mRNA levels 2 and 5 DIV after hNolz over-expression or silencing with Nolz1 siRNA in NPCs (Figure 11A). We did not detect $R A R \gamma$ expression in control NPCs, or after transfection of hNolz or Nolz1 siRNA (data not shown). In contrast, both $R A R \alpha$ and $R A R \beta$ were highly expressed in NPCs. $R A R \alpha$ levels were not modified in any condition studied whereas RAR $\beta$ levels were increased 5 DIV after $h N o l z$ over-expression and reduced after Nolz1 silencing (Figure 11A). In addition, $h N o l z$ over-expression did not regulate the level of Raldh3 mRNA (Figure 11A). Similarly, the levels of CRBP1 and Cyp26, two other limiting proteins for RA metabolism, were not modified by hNolz overexpression (data not shown). These findings indicate that Nolz1 expression does not increase RA levels but changes the competence of cells to respond to RA. To further test this hypothesis, we analyzed whether hNolz over-expression could activate RA signaling using a Luciferase RARE-reporter assay. Three DIV after transfection, an increase in luciferase activity was observed (Figure 11B), demonstrating that this transcription factor leads to an increase in RA signaling. These results suggested that Nolz1 could induce the conversion 

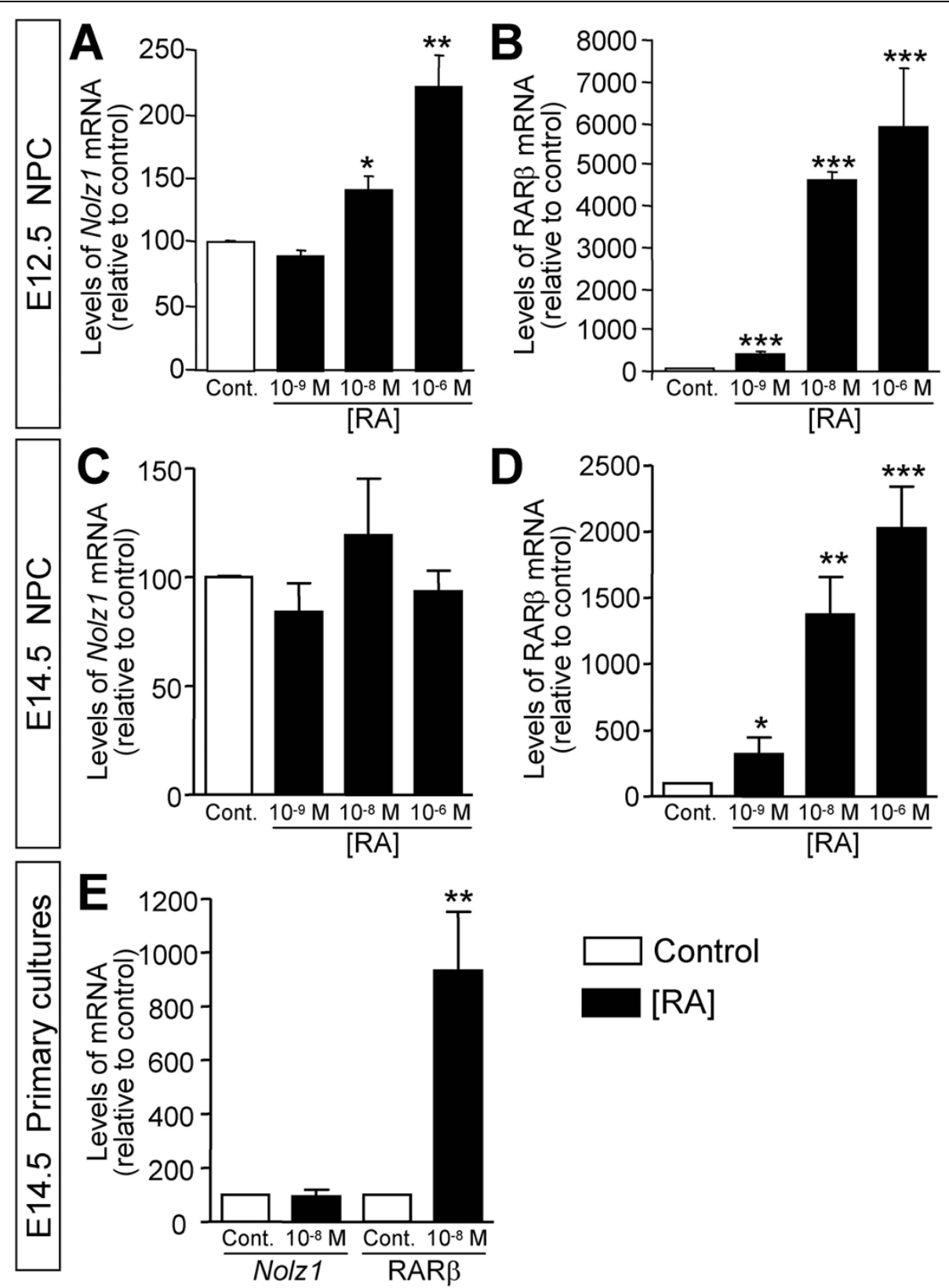

Figure 8 Striatal cultures are temporarily competent to regulate Nolz1 expression in response to RA. NPC and primary cultures were treated with RA and the expression of Nolzl and RAR $\boldsymbol{\beta}$ were analyzed by Q-PCR. (A,B) RA increases the expression of Nolz1 and RAR $\boldsymbol{\beta}$ in a dosedependent manner $\left(0,10^{-9}, 10^{-8}\right.$ and $\left.10^{-6} \mathrm{M}\right)$ in E12.5-derived neurospheres. $(\mathbf{C}, \mathbf{D})$ Within E14.5-derived neurospheres, RA increases the expression of $R A R \beta$ in a dose-dependent manner (D) but it does not affect Nolz1 mRNA levels (C). (E) RA increases the levels of RAR $\beta$ without affecting Nolz1 mRNA levels in LGE primary cultures treated with $10^{-8} \mathrm{M}$ RA during 3 DIV. The results represent the mean \pm standard error of the mean from at least three independent samples at each condition. Statistical analysis was performed with one-way ANOVA, followed by the Bonferroni post-hoc test (A-D) or with the Student's $t$-test $(\mathrm{E}) .{ }^{*} P<0.05,{ }^{* *} P<0.01,{ }^{* * *} P<0.001$ relative to control.

of NPCs into neuronal cells through the regulation of RAR $\beta$-mediated signaling. Thus, we next analyzed whether Nolz1 mediates NPC cell cycle exit and promotes neuronal differentiation by this mechanism. Surprisingly, RA signaling does not seem to mediate Nolz1-regulated proliferation of NPCs, since under the same conditions that $h N o l z$ modified BrdU incorporation, treatment with RA (Figure 12A) or a RAR $\beta$-specific agonist (Figure 12B) did not affect neurosphere proliferation (Figure 12). Thus, we next analyzed whether RA signaling was necessary for Nolz1-induced neurogenesis. To this end, we transfected primary striatal cultures 


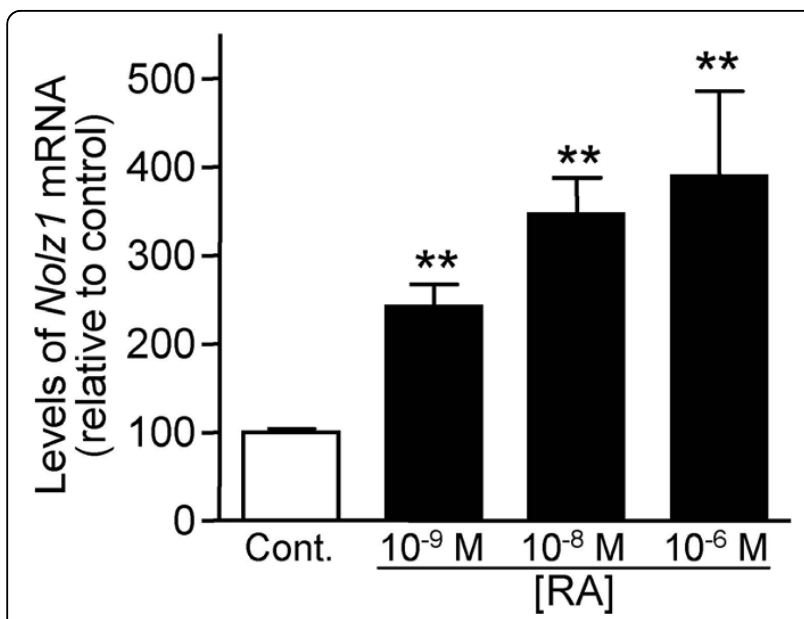

Figure 9 Nolz 1 expression is induced by RA in embryoid bodies derived from mouse embryonic stem cells. Embryoid bodies were treated with various concentrations of RA $\left(0,10^{-9}, 10^{-8}\right.$ and $\left.10^{-6} \mathrm{M}\right)$ and 4 DIV later Nolz1 expression levels were analyzed by Q-PCR. Values are normalized to control (without RA), considered as $100 \%$, and expressed as the mean from at least three independent samples at each stage studied. Error bars represent the standard error of the mean. Statistical analysis was performed with one-way ANOVA, followed by the Bonferroni post-hoc test. ${ }^{*} P<$ 0.01 relative to control.

with $h N o l z$ in the absence of RA, since cells were cultured in a RA-free medium supplemented with 4-diethylaminobenzaldehyde (DEAB), a Raldh inhibitor [33]. Under these conditions, the levels of $R A R \beta$ and Raldh 3 were highly reduced (Figure 13A). Moreover, hNolz could not induce neuronal differentiation in the absence of RA, since hNolz-induced neurogenesis was lost in the presence of DEAB (Figure 13B,C). To further study whether the effect of Nolz1 in neurogenesis was not only dependent on RA but also on RAR $\beta$ signaling, we next over-expressed $h N o l z$ in striatal primary cultures treated with a pan-RAR inverse agonist (BMS493, also named UVI2024, [34,35]). In these conditions, hNolz was also unable to induce an increase of Tuj1-positive cells and a reduction of nestin-positive precursors or GFAP-positive astroglia (Figure 14). Therefore, these results demonstrate that Nolz1 needs RA signaling through RARs to mediate striatal neurogenesis. However, the stimulation of RAR $\beta$ with a specific agonist (BMS641, also named UVI2003, [36]) was not sufficient to induce changes in neural markers in striatal cultures (Figure 15).

\section{Tle4 and Nolz1 have parallel expression patterns in both} the LGE in vivo and LGE-derived NPCs

Due to the ability of the NET family of transcription factors, which includes Nolz1, to bind to members of the Gro-TLE family of transcriptional repressors
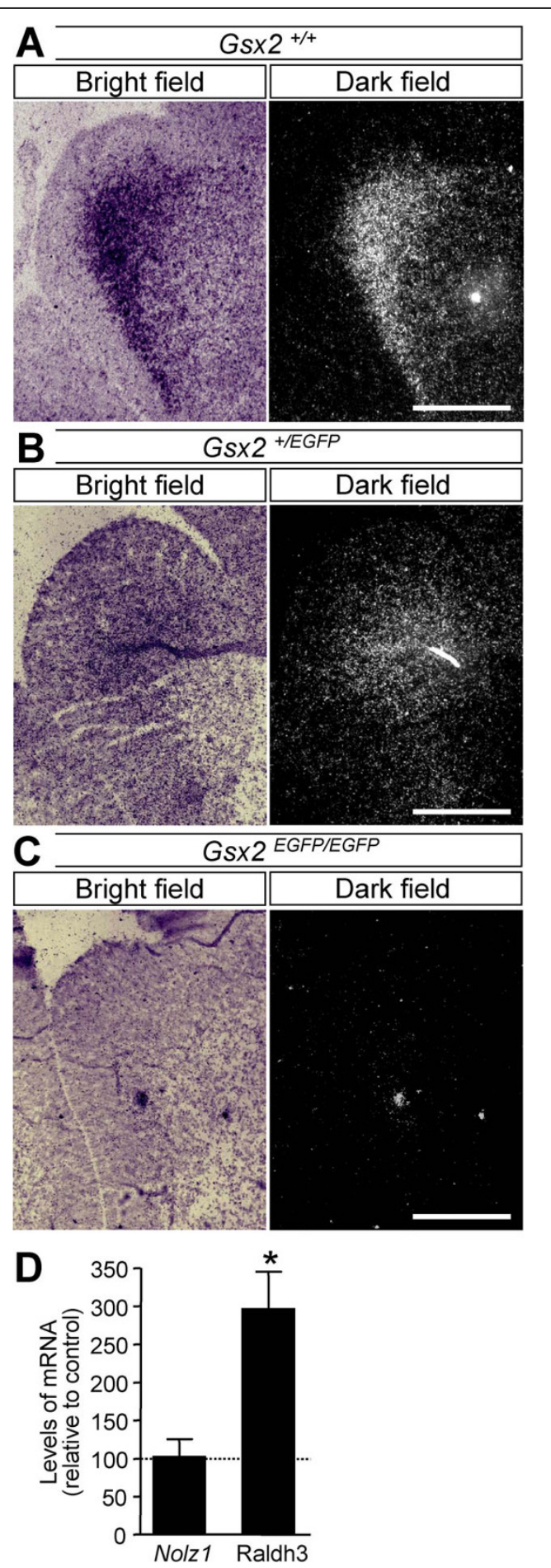

Figure $10 \mathrm{Gsx} 2$ is a permissive factor for Nolz1 expression. (A-C) Nolz1 mRNA expression is detected in E14.5 wild-type mice $\left(G s \times 2^{+/+} ; A\right)$ and Gsx2 $2^{+/ E G F P}$ mice $(B)$, but totally disappears in the vLGE of GsX2 $2^{E G F P / E G F P}$ mice (C). Scale bars: $600 \mu \mathrm{m}$. (D) Gsx2 overexpression in NPCs does not modify Nolz1 mRNA levels, although it increases Raldh3 mRNA expression. The results represent the mean \pm standard error of the mean from at least three independent samples at each condition. Statistical analysis was performed with the Student's $t$-test. ${ }^{*} P<0.05$, relative to control. 


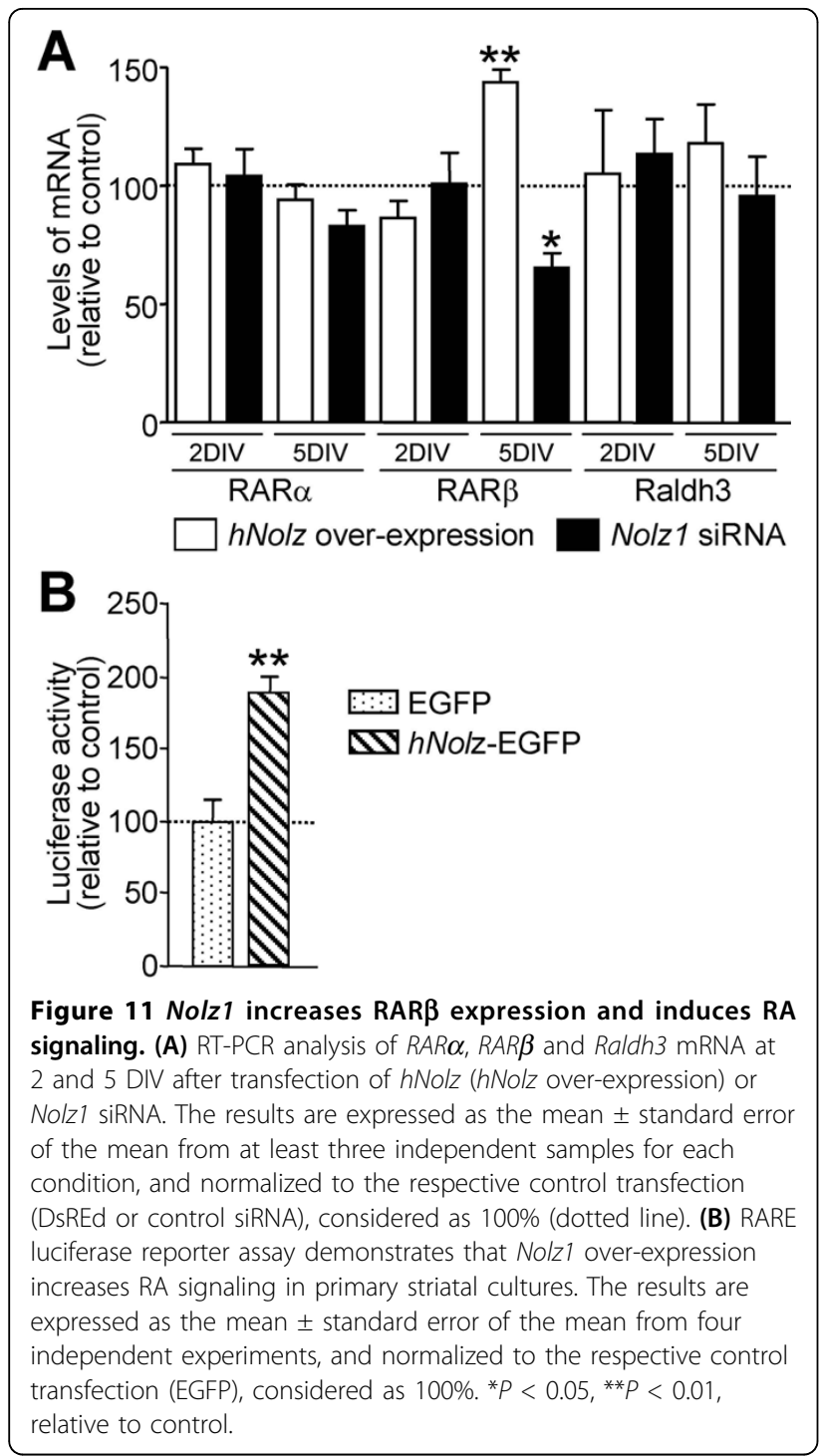

$[22,20,24]$, we analyzed the expression of several members of the Gro-TLE family in the LGE at E14.5 by in situ hybridization (Figure 16). We did not detect any Tle 2 expression, and Tle3 mRNA was slightly but broadly expressed through the telencephalon (Figure 16A). Tle1 and Tle4, in contrast, were specifically regulated in the different proliferative zones of the LGE (Figure 16A). Tle1 expression was maximal in the VZ, and decreased in the SVZ and MZ (Figure 16A). In contrast, Tle4 was not expressed in the $\mathrm{VZ}$ and its levels were high in the SVZ, extending to the MZ but at lower expression levels (Figure 16A), thus resembling the expression pattern of Nolz1 (Figure 2). To determine if Nolz1 and Tle4 were co-expressed in the LGE, we performed in situ hybridization for Nolz1 followed by immunohistochemistry for Tle4, which confirmed their colocalization within the LGE (Figure 16B). We also

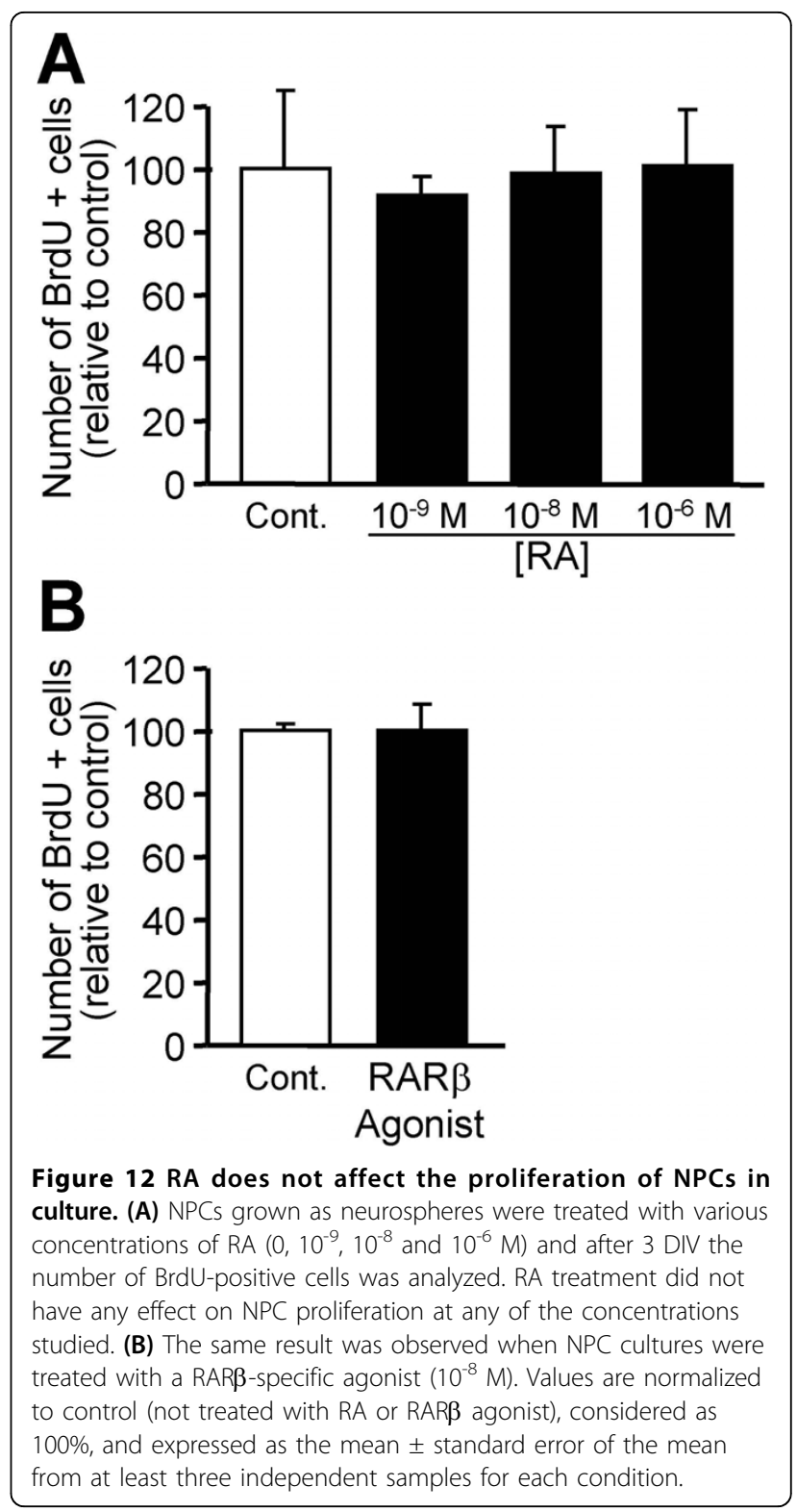

analyzed the Tle4 expression pattern during NPC differentiation. Tle4 followed a pattern of expression similar to that of Nolz1, as its maximal levels were achieved in non-differentiated NPCs and decreased with differentiation (Figure 16C).

\section{Discussion}

Our results show that Nolz1 expression is high in the SVZ and is maintained at low levels in the MZ of the LGE, suggesting a dual effect promoting cell cycle exit and neuronal differentiation of NPCs. We found that Nolz1 expression negatively regulates the proliferation and induces cell cycle exit of NPCs cultured as neurospheres. In addition, in LGE-derived primary neural cultures, Nolz1 over-expression increased the number of 

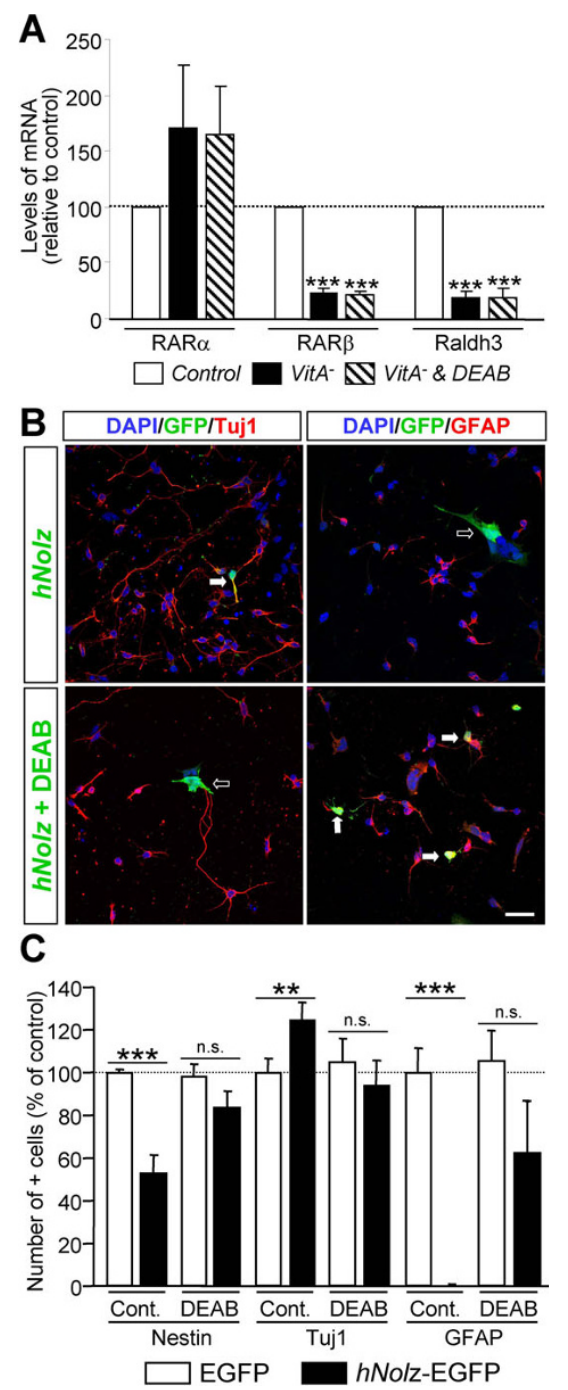

Figure 13 Nolz1-mediated neurogenesis depends on RA signaling. (A) RT-PCR analysis of $R A R \alpha, R A R \beta$ and Raldh 3 mRNA expression in NPC cultures grown in normal conditions (control), in vitamin $A$-free medium (Vit $\mathrm{A}^{-}$) and in vitamin A-free medium in the presence or DEAB (VitA \& DEAB) demonstrates a decrease in $R A R \beta$ and Raldh3 mRNA in the absence of RA. The results are expressed as the mean \pm standard error of the mean from at least three independent samples for each condition, and normalized to the control medium, considered as 100\%. Statistical analysis was performed with the Student's $t$-test. ${ }^{* *} P<0.001$ relative to control. $(\mathbf{B}, \mathbf{C})$ Nolz1 does not induce neuronal differentiation in a RA-free environment. (B) Representative pictures of immunofluorescence for Tuj1 or GFAP after transfection of hNolz in the absence or presence of DEAB. White arrows show double positive cells, open arrows show single EGFP stained cells. Scale bar: $30 \mu \mathrm{m}$. (C) Treatment with $1 \mu \mathrm{M}$ DEAB in a vitamin A-free medium (DEAB) impairs the increase of Tuj1 and the reduction of GFAP observed in hNolz-transfected primary striatal cultures in control conditions (Cont.). The results are expressed as the mean \pm standard error of the mean from at least three independent samples for each condition, and normalized to the control medium, considered as $100 \%$. Statistical analysis was performed with the Student's t-test (C). ${ }^{* *} P<0.01$, ${ }^{* * *} P<0.001$ relative to control. N.s., not significant.
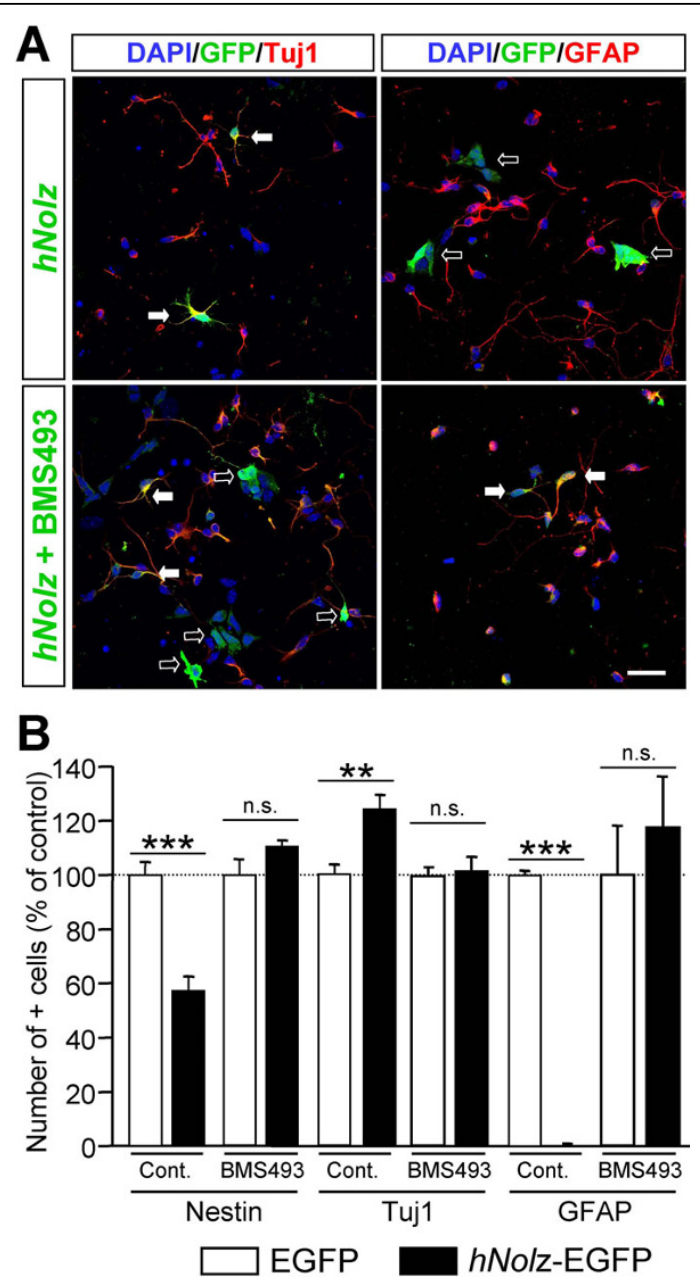

Figure 14 RAR activity is essential for Nolz1-mediated neurogenesis. (A,B) Nolzl does not induce neuronal differentiation in the presence of a RAR inverse agonist. (A) Representative pictures of immunofluorescence for Tuj1 or GFAP after transfection of hNolz in the absence or presence of a RAR inverse agonist (BMS493).

White arrows show double positive cells, open arrows show single EGFP stained cells. Scale bar: $30 \mu \mathrm{m}$. (B) The presence of the RAR inverse agonist (10-8 M BMS493) impairs the increase of Tuj1 and the reduction of GFAP observed after hNolz transfection in primary striatal cultures (Cont.). The results are expressed as the mean \pm standard error of the mean from at least three independent samples for each condition, and normalized to the transfection in standard culture conditions (Cont.), considered as 100\%. Statistical analysis was performed with the Student's $t$-test. ${ }^{* *} P<0.01,{ }^{* *} P<$ 0.001 relative to control.

neurons at the expense of nestin- and GFAP-positive cells, indicating that Nolz1 participates in striatal neurogenesis. We also show that Nolz1-induced neurogenesis depends on RA signaling, since this transcription factor promoted the expression of RAR $\beta$ in LGE-derived NPCs and its neurogenic effect was impaired in a RA-signaling-free context.

The shape of the telencephalon and the relative sizes and positions of its cell populations result from 


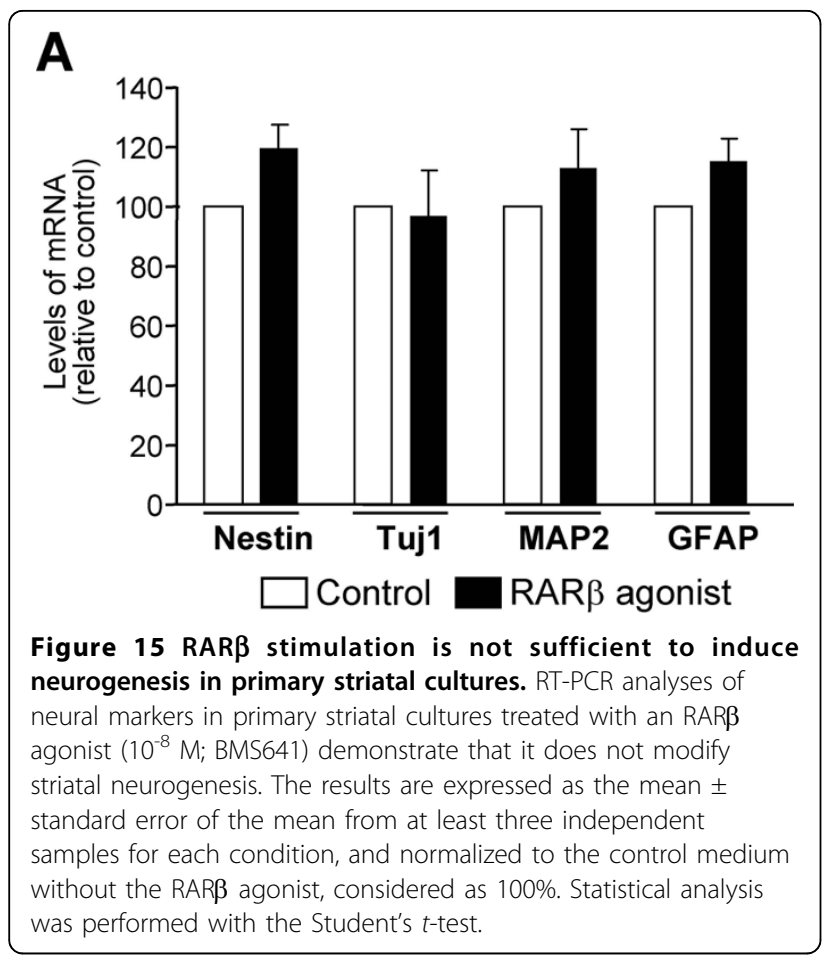

variations in cell proliferation [37] and many transcription factors have important roles in this mechanism. Our results show that Nolz1 contributes to the homeostasis of NPCs in the SVZ of the VLGE, where it is highly expressed ([19] and our present results), by reducing the proliferation, promoting cell cycle exit and possibly reducing the self-renewal potential of NPCs. Cell cycle exit during LGE embryonic development is usually associated with proneural transcription factors. Here we demonstrate that Nolz1 promotes the acquisition of a neuronal phenotype since over-expression of Nolz1 in LGE primary cultures produces an increase in the number of neurons. Proneural factors such as Ascl I (also known as Mash1) [32] are restricted to the VZ and SVZ, where progenitors begin to differentiate, but are absent in the MZ, where fully differentiated neurons are found. However, other transcription factors such as Meis 2 or Islet are expressed in both the germinal VZ and SVZ and in the MZ [28,38]. Similarly, Nolz1 expression is high in the SVZ and its expression decreases but does not disappear in the MZ, where striatal neurons continue to express low levels of Nolz1 ([19] and present results). These results agree with the idea that stable specification of cell identity requires mechanisms that maintain patterns of gene expression over long periods of time [39].

One of the main issues in cell fate specification is the understanding of the interaction between two general sets of determinative factors: secreted extrinsic signals present in a cell's local environment and intrinsic signals that operate in a cell-autonomous manner [39]. Keeping with this view, RA signaling can increase Nolz1 levels in the PC12 cell line [19] and in chick postmitotic motoneurons [20]. Our results demonstrate that Nolz1 expression was not modified when a vitamin A-deficient diet was used to feed the mothers, which results in lowered levels of RA synthesis in the pups throughout the whole of development [31]. In addition, Nolz1 expression was not affected in Raldh3-deficient animals, which have been shown to lack RA activity in the LGE [11]. These findings suggest that the levels of RA are not limiting for Nolz1 expression. Reinforcing this idea, we observed that RA does not regulate Nolz1 expression in E14.5-derived NPCs or primary cultures. However, E12.5-derived NPCs do respond to RA by increasing the expression of Nolz1. Similarly, RA was able to regulate Nolz1 in mouse embryonic stem cells. Altogether, these data indicate that the ability of cells to induce Nolz1 in response to RA is dynamically regulated during striatal development. Thus, a very interesting possibility is that RA participates in the induction but not in the maintenance of Nolz1 expression, since other sources of RA in the LGE has been described [40].

Interestingly, we found that Gsx 2 is required for the correct expression of Nolz1 in the vLGE, the mouse telencephalic structure that gives rise to striatal projection neurons (for a review, see [41]). In agreement with this result, it has recently been shown that Gsx2 participates in the temporal specification of neuronal fate in the LGE [42]. The development of striatal projection neurons depends on early actions of Gsx2 during differentiation of the vLGE, where Nolz1 is expressed, while differentiation of the dLGE, where Nolz1 is not expressed, requires Gsx2 function at least until birth [42]. However, over-expression of Gsx2 in NPCs did not modify the levels of Nolz1, suggesting that Gsx2 acts as a permissive instead of an inductive factor for Nolz1 expression. Since Gs $x 2$ is involved in the differentiation of striatal projecting neurons through the regulation of RA signaling [18], Nolz1 could act downstream of Gs $x 2$ to regulate RA signaling. We found that Nolz1 regulates the expression of RAR $\beta$, the RA receptor that has been shown to promote striatal projection neuron differentiation $[12,15,43]$. In addition, early striatal neurogenesis is affected in RAR $\beta$ mutant mice [44], coinciding with the expression and function of Nolz1. In fact, Nolz1 and $R A R \beta$ are expressed in the MZ at the same developmental stages ([19,45] and present results). Furthermore, our present results demonstrate that Nolz1 induces not only increases in $R A R \beta$ but also RA signaling, which is essential to exert its neurogenic effect in primary striatal cultures. Interestingly, although this receptor is necessary for Nolz1-induced neurogenesis, its activation by a 

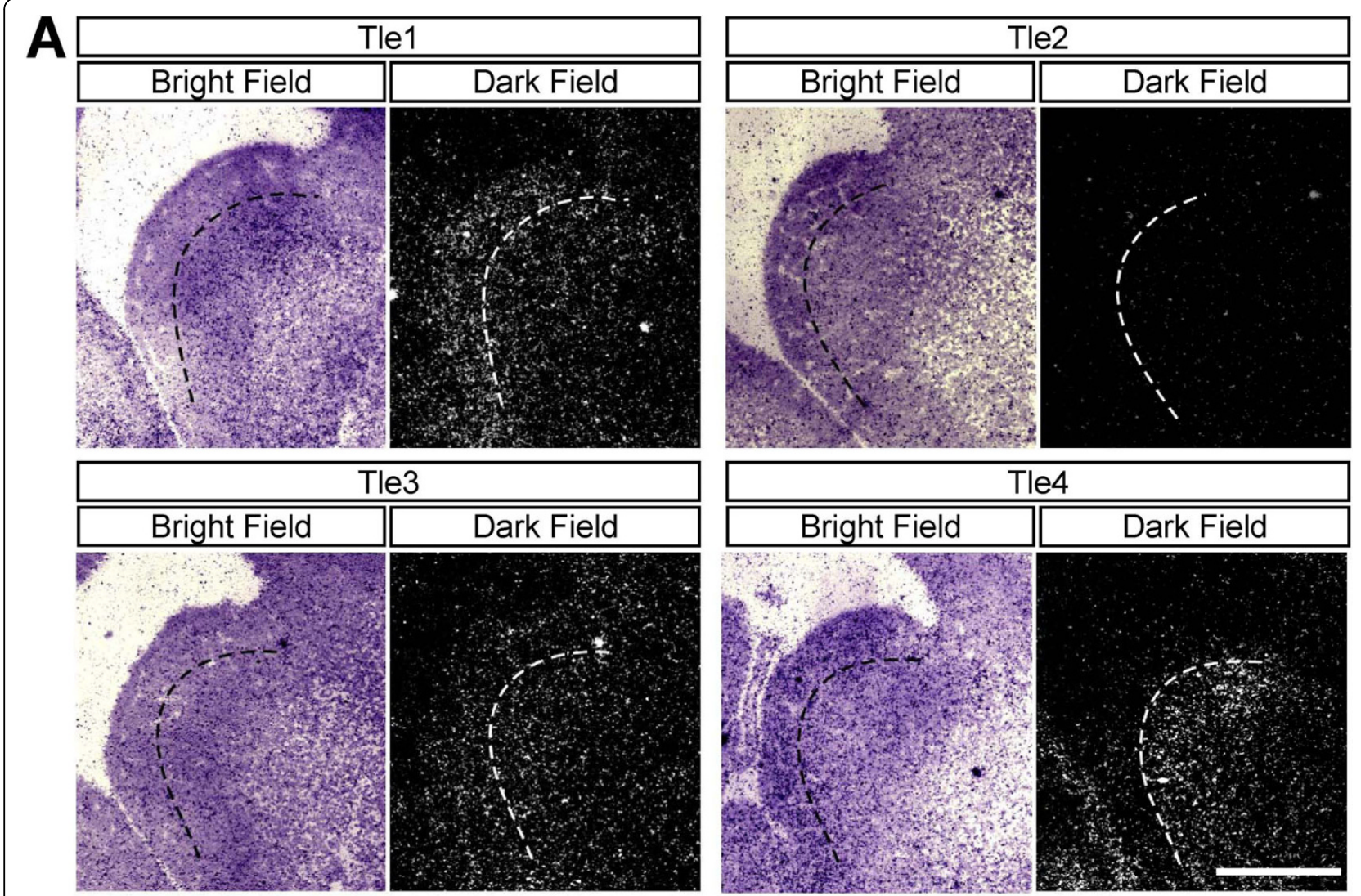

B
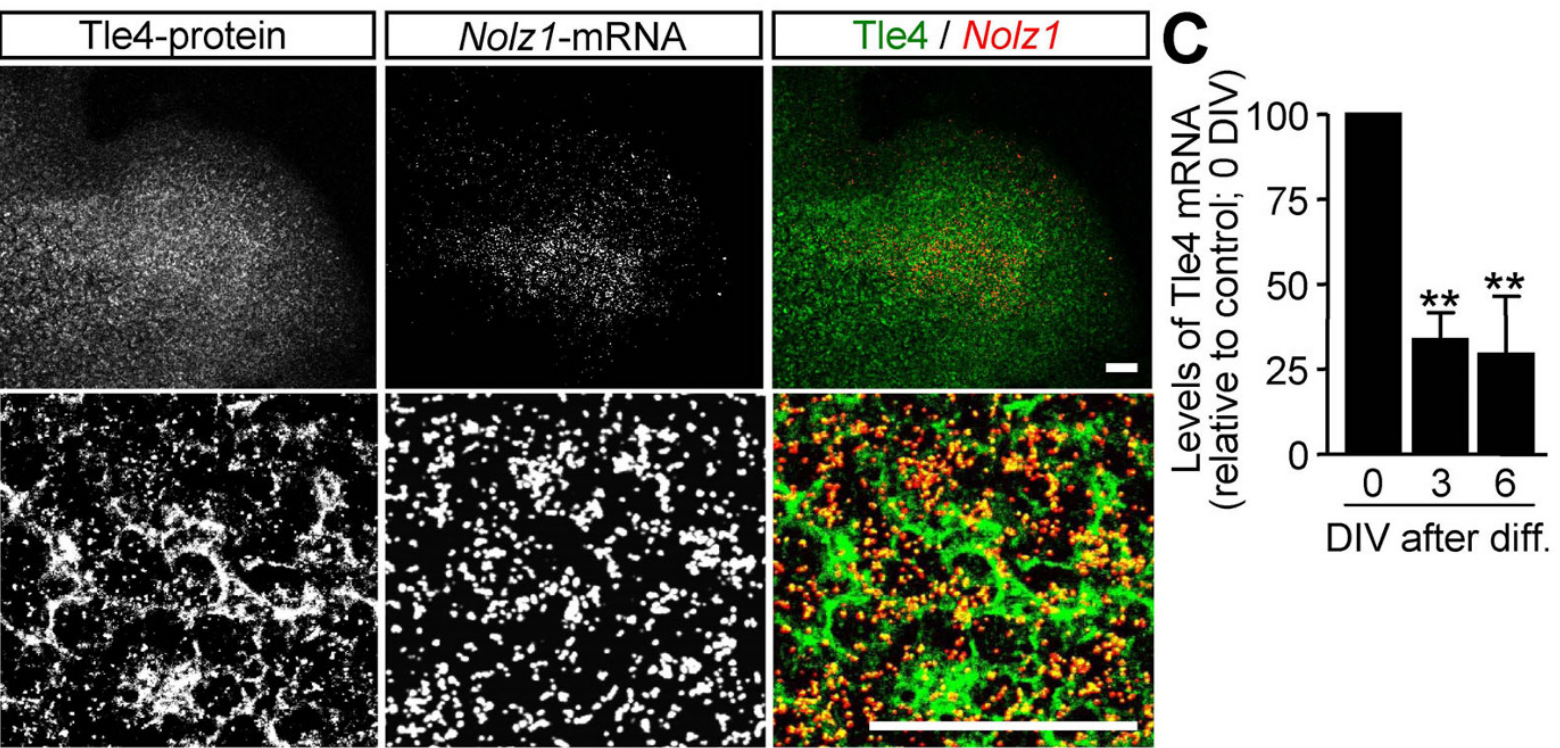

Figure 16 Tle4 and Nolz1 expression show similar patterns in the developing LGE and in NPCs. (A) In situ hybridization study to analyze Tle1, Tle2, Tle3 and Tle4 mRNA levels in the LGE at E14.5. Dashed lines mark the VZ-SVZ boundary. (B) Simultaneous Tle4 immunohistochemistry and Nolz1 in situ hybridization show coincident expression patterns in the LGE at E14.5. (C) High levels of Tle4 mRNA are observed in proliferating NPCs (0 DIV), which decrease after differentiation ( 3 and 6 DIV). The results are expressed as the mean from at least three independent samples for each condition, and normalized to the proliferating NPCS (0 DIV), considered as 100\%. Error bars represent the standard error of the mean. Statistical analysis was performed with one-way ANOVA, followed by the Bonferroni post-hoc test. ${ }^{* *} P<0.01$ relative to control. Scale bars: $600 \mu \mathrm{m}(\mathrm{A}) ; 100 \mu \mathrm{m}$ (B). 
selective RAR $\beta$ agonist is not sufficient to stimulate any changes in neural markers. This result suggests that Nolz1 may also act on additional pathways to promote neural differentiation. Altogether, these findings show that Nolz1 participates in the early Gsx2-dependent differentiation of vLGE-derived neurons through the regulation of RAR $\beta$-mediated signaling.

It has been shown that Gsx2 mutant mice have reduced levels of Raldh3 [18]. In addition, we show that over-expression of Gs $x 2$ in NPCs increases the mRNA levels of this striatal RA-limiting enzyme. However, we observed that Nolz1 did not regulate the expression of Raldh3, so it is not regulating RA synthesis. These results suggest that Gs $x 2$ may regulate RA signaling at two different levels; one is Nolz-independent and mediated by the regulation of Raldh 3 and the other involves the regulation of Nolz1 expression, which in turn regulates the levels of $R A R \beta$.

$R A R \beta$ is expressed in the MZ while $R A R \alpha$ is broadly expressed in the VZ and SVZ through the entire telencephalon and is important in the control of the proliferation of neural precursors $[11,46]$. We observed that $R A R \alpha$ is unaffected by Nolz1 over-expression. Thus, it seems that Nolz1 acts through RA signaling to regulate the differentiation of striatal projection neurons but its effect on NPC proliferation is independent of RA. In fact, we observed that RA did not modify the proliferation of NPCs in vitro. Increases in cell cycle length could lead to progressive restriction of the proliferation potential of LGE NPCs, and it can be promoted by transcription factors such as Foxg1 [37]. However, Nolz1 did not induce changes in cell cycle length. Another interesting possibility is that Nolz1 and Gsx2 function together to regulate proliferation since Gsx 2 expression in the SVZ coincides with Nolz1 $([19,32]$ and present results). Keeping with this view, it has been shown that the Gsx2 homolog in Drosophila, Ind (intermediate neuroblasts defective), interacts with Gro proteins, which are able to bind elB (elbow B), the Nolz1 homolog, acting as transcriptional co-repressors [27]. In addition, the mammalian Nolz1 protein also contains the conserved FKPY sequence, which allows binding to Gro-TLE proteins (Figure 1A). We observed that many TLE (NocAelB-Tlp) proteins, the mammalian homologs of Gro, are expressed in the vLGE. Among them, TLE4 follows a similar expression pattern to Nolz1, with its highest levels in the SVZ, while the VZ is enriched in TLE1 expression. These results are similar to those observed in the cerebral cortex, where TLE4 is expressed by more differentiated NPCs of the SVZ and TLE1 expression is elevated in the undifferentiated VZ neural precursors [47-49]. Moreover, TLE4 expression decreased upon NPC differentiation. Taken together, these findings suggest that Nolz1 and TLE4 could act together to control
SVZ proliferation in the LGE. Similarly, it has been recently described that Nolz1 requires, in part, the modulatory activity of Grg5, an atypical member of the GroTLE family of co-repressors, to control motor neuron determination in the chick spinal cord [20]. However, further experiments are required to analyze functional interactions between these proteins.

In conclusion, we demonstrate that Nolz1 has a dual effect on NPCs, on one hand by controlling their proliferation and promoting cell cycle exit, and on the other by inducing striatal neurogenesis. Nolz1 over-expression increases the number of striatal neurons downstream of Gs $x 2$ by inducing RA signaling through RARs. Its properties and expression pattern suggest that the activity of Nolz1 in the vLGE could be modulated by co-repressors, such as Gro-TLE.

\section{Materials and methods \\ Animal subjects}

All animals were housed with access to food and water ad libitum in a colony room maintained at a constant temperature $\left(19\right.$ to $22^{\circ} \mathrm{C}$ ) and humidity (40 to $\left.50 \%\right)$ on a 12:12 h light:dark cycle. Animal treatments and handling procedures were approved by the Local Committees, in accordance with the European Community Council Directive (86/609/EU).

B6CBA wild-type mice (from Charles River Laboratories, Les Oncins, France), Raldh3-deficient mice [11], and Gs $x 2$-deficient mice [32] were used in this study. For embryonic ages, time of pregnancy was determined by first detection of a vaginal sperm plug by daily inspection and considered as E0.5. For postnatal studies, the day of birth was considered as postnatal day 0 (P0).

To induce vitamin A deficiency in mice, pregnant mice were fed with the vitamin A-deficient diet TD.86143 (Harlan Laboratories Inc., Indianapolis, IN, USA).

\section{Culture procedures}

E12.5 or E14.5 fetal brains were excised and placed in sterile phosphate-buffered saline $\mathrm{pH}$ 7.4, and the LGEs were dissected bilaterally, pooled and gently dissociated with a fire-polished Pasteur pipette.

Mixed neuron-glial primary cultures were obtained by plating the cells onto 24-well plates containing glass coverslips precoated with $0.1 \mathrm{mg} / \mathrm{ml}$ poly-D-lysine (Sigma Chemical Co., St Louis, MO, USA) at a density of 150,000 cells $/ \mathrm{cm}^{2}$ in Eagle's minimum essential medium (Invitrogen SA, Prat de Llobregat, Barcelona, Spain) supplemented with $7.5 \%$ fetal bovine serum (FBS; Invitrogen SA), 0.6\% D-(+)-glucose (Sigma Chemical Co.), $100 \mathrm{U} / \mathrm{mL}$ of penicillin and $100 \mathrm{mg} / \mathrm{mL}$ streptomycin (both obtained from Invitrogen SA). Three or 5 days after seeding, cultures were fixed with $4 \%$ 
paraformaldehyde solution (PFA; Merck Biosciences Ltd, Nottingham, UK) in $0.1 \mathrm{M}$ phosphate buffer $\mathrm{pH} 7.4$ and processed for immunocytochemistry.

LGE-derived neurosphere cultures were obtained by seeding 50,000 cells $/ \mathrm{cm}^{2}$ in medium containing Dubelcco's Modified Eagle's Medium (DMEM; Sigma Chemical Co.):F12 (Invitrogen SA) (1:1); supplemented with 0.3\% glucose (Sigma Chemical Co.), $0.3 \mathrm{mg} / \mathrm{ml}$ glutamine (Invitrogen SA), 5 mM HEPES (Invitrogen SA), $100 \mathrm{U} /$ $\mathrm{ml}$ penicillin, and $100 \mathrm{mg} / \mathrm{ml}$ streptomycin (Invitrogen SA), $4 \mu \mathrm{g} / \mathrm{ml}$ heparin (Sigma Chemical Co.), $4 \mathrm{mg} / \mathrm{ml}$ bovine serum albumin (Sigma Chemical Co.), $1 \times \mathrm{N} 2$ supplement (Invitrogen SA), $20 \mathrm{ng} / \mathrm{ml}$ fibroblast growth factor (Sigma Chemical Co.) and $10 \mathrm{ng} / \mathrm{ml}$ epidermal growth factor (Invitrogen SA). Every 5 days neurospheres were collected, dissociated by pipetting approximately 40 times with a P100 micropipette and re-plated in fresh media at a density of 10,000 cells $/ \mathrm{cm}^{2}$.

For cell differentiation, 12,500 cells $/ \mathrm{cm}^{2}$ were seeded. The day after plating, cells were collected and incubated onto Matrigel-treated coverslips (Growth Factor Reduced Matrigel Matrix, BD Biosciences, ClontechTakara Bio Europe, Saint-Germain-en-Laye, France). Media was changed to medium supplemented with only $20 \mathrm{ng} / \mathrm{ml}$ fibroblast growth factor (Sigma Chemical Co.) and cells were allowed to differentiate in this medium for 2 more days. Then, media was changed again to medium supplemented with $2 \%$ FBS (Invitrogen SA) and cells were grown for 3 more days (until a total of 6 days of in vitro differentiation). Cell pellets for each time point ( 0,3 and 6 days of differentiation) were obtained and frozen at $-80^{\circ} \mathrm{C}$ for RNA or protein extraction.

In the present study, we used the mouse embryonic stem cell line R1 obtained from Dr Andras Nagy's laboratory. The maintenance of undifferentiated mouse embryonic stemcells, embryoid body formation and culture were carried out as previously described [15].

All cell cultures were incubated at $37^{\circ} \mathrm{C}$ in a $5 \% \mathrm{CO}_{2}$ atmosphere.

\section{RA and RAR $\beta$ agonist treatment}

Neurospheres were passaged as described above and single cells were seeded in 6-well plates at a density of 100 cells $/ \mathrm{mm}^{2}$ with fresh culture medium containing different concentrations of all-trans-RA $\left(10^{-9}, 10^{-8}\right.$ and $10^{-6} \mathrm{M}$; Sigma Chemical Co.) dissolved in dimethyl sulfoxide (DMSO; Sigma Chemical Co.). Control cells were cultured with the same dilutions of the RA vehicle, DMSO. Some cultures were treated with a RAR $\beta$-specific agonist [36] at a concentration of $10^{-8} \mathrm{M}$ dissolved in DMSO, which we observed is effective in these cultures ( $\mathrm{R}$ Martín-Ibáñez et al., in preparation). NPCs were allowed to grow for 3 DIV and then were pelleted for RNA extraction and RT-PCR analysis or processed for BrdU immunocytochemistry.

Mixed neuron-glial LGE primary cultures were grown for 3 DIV and RA or RAR $\beta$ agonist (BMS641) dissolved in DMSO was added to the medium at the concentration of $10^{-8} \mathrm{M}$. Fresh RA/RAR $\beta$ agonist was added every 24 hours of culture and 3 hours prior to fixation.

Embryoid bodies were formed as described above and RA was added to the culture medium at different concentrations $\left(10^{-9}, 10^{-8}\right.$ and $\left.10^{-6} \mathrm{M}\right)$. They were grown for 4 DIV and then pelleted for RNA extraction and RT-PCR analysis.

\section{Cell transfection}

To over-express NolzI, cells were transfected with the pNolz-IRES2-DsRED plasmid, that was obtained by clonation of the human NolzI contained in the pOTB plasmid (MGC full-length (IRAU) collection, clone ID 4053098) into EcoRI and SmaI restriction sites of pIRES2-DsRED-Express plasmid (BD Biosciences) coding for DsRED fluorescent protein. As a control, we used pIRES2-DsRED-Express empty plasmid. Tranfection was made with $9 \mu \mathrm{g}$ of NolzI-RED or RED plasmid.

To reduce NolzI expression, three different siRNAs against NolzI mRNA were used (Silencer Pre-designed siRNAs, IDs 89661, 169777, 89565, Ambion, Applied Biosystems, Foster City, CA, USA). Tranfection was made with $2 \mu \mathrm{M}$ of each siRNA or $6 \mu \mathrm{M}$ of negative control siRNA (Silencer Negative Control \#1 siRNA, Ambion).

Low passage (four to seven) embryonic neurospheres were disaggregated and transfected by nucleofection following the manufacturer's protocol (Amaxa Biosystems, Lonza Iberica SA, Barcelona, Spain). Using the A33 Nucleofector program (Amaxa Biosystems), $5 \times$ $10^{6}$ cells were transfected. Viable cells were counted by trypan blue exclusion in a Neubauer chamber after nucleofection.

For BrdU incorporation assays, 65,000 cells $/ \mathrm{cm}^{2}$ were seeded after nucleofection. Two days later cells were collected and incubated for 10 minutes in 24-well plates with Matrigel-treated coverslips and they were incubated for 10 minutes in BrdU-containing media at a final concentration of $2 \mu \mathrm{g} / \mathrm{ml}$. Just after incubation, cells were fixed and processed for immunocytochemistry.

The cell cycle exit index was analyzed as described elsewhere [30]. Briefly, we performed the same procedure as for BrdU incorporation except that neurospheres were pulsed with BrdU the same initial day of inducing hNolz over-expression and cells were fixed 3 days later. Thereafter, neurosphere cultures were processed for BrdU and Ki67 immunostaining. BrdU-positive but Ki67-negative cells were counted as the cells that left the cell cycle during the experimental period. 
In order to analyze cell cycle duration, 10 neurospheres were plated 3 days after transfection in 96-well plates with complete medium and supplemented with 1 $\mu \mathrm{M}$ BrdU. Neurospheres were attached at 1, 3, 6, 12 and 24 hours after treatment in 96 well-plates pre-coated with Matrigel. Ten minutes later, cells were fixed in $4 \%$ PFA and processed for immunocytochemistry.

For the self-renewal assay, 65,000 cells $/ \mathrm{cm}^{2}$ were seeded after nucleofection and the total number of neurospheres obtained 5 days later was counted (passage 0 (P0) after transfection). Cells were dissociated and 2,500 cells $/ \mathrm{cm}^{2}$ were seeded and counted again on day 5 (passage 1 (P1) after transfection).

For differentiation after nucleofection, 50,000 cells/ $\mathrm{cm}^{2}$ were seeded and the same protocol as for nonnucleofected cells was followed.

To over-express Nolz1 in primary cultures, we transfected the cells with the pLV-Nolz-IRES-EGFP plasmid or the pLV-IRES-EGFP plasmid, which encode $h N o l z$ and EGFP or EGFP only, respectively. The pLV-IRESEGFP plasmid was generated using the pRRLsinPPT plasmid (pRRL) constructed by the Miami Project to Cure Paralysis Viral Vector Core Lab based on the lentiviral transducing plasmid developed by Naldini et al. [50]. Briefly, the multiple cloning site (MCS) of the pRRL plasmid was substituted by the MCS-IRES-EGFP from the PRV-IRES-EGFP (Genetrix SL, Tres Cantos, Madrid, Spain) using the BamHI and the SalI restriction sites. To construct the pLV-Nolz-IRES-EGFP, the hNolz gene from the pNolz-IRES2-DsRED plasmid was cloned into pLV-IRES-EGFP between the MCS BamHI and XhoI sites. Primary cultures were transfected 24 hours after seeding with $0.5 \mu \mathrm{g}$ of the corresponding plasmids per well (24-well plate). The transfection was performed using Lipofectamine LTX (Invitrogen SA), following the manufacturer's instructions. Three days after transfection cells were fixed with 4\% PFA for immunocytochemistry analysis.

For DEAB (Sigma Chemical Co.) treatment, primary cultures were transfected $12 \mathrm{~h}$ after seeding as described above. Then, $24 \mathrm{~h}$ later DEAB was added to the medium at a concentration of $10^{-8} \mathrm{M}$. Three days after treatment cells were fixed with $4 \%$ PFA for immunocytochemistry analysis.

For RAR inverse agonist administration, primary cultures were transfected $12 \mathrm{~h}$ after seeding as described above. Thereafter, the RAR inverse agonist (BMS493 [35]) was added to the medium at a concentration of $10^{-8} \mathrm{M}$ in DMSO, which was repeated every single day. At 3 DIV cells were fixed with $4 \%$ PFA for immunocytochemistry analysis.

\section{Production of viral particles and cell transduction}

To over-express Gsx2, the human Gsx2 gene from the pcDNA-hGsx2 plasmid, kindly provided by Dr Peter Marynen (Université de Leuven, Belgium), was PCRcloned into the retroviral vector pRV-IRES-EGFP using the MCS BamHI and XhoI sites.

For retrovirus production, 293T cells were plated at a density of approximately $6 \times 10^{4}$ cells per $\mathrm{cm}^{2}$. The following day, cells were transfected by a three-plasmid system (the pRV-Gsx2-IRES-GFP plasmid, the plasmid that expresses HIV-1 gag and pol genes, and the plasmid that expresses vesicular stomatitis virus $\mathrm{G}$ ) using the calcium phosphate/DNA co-precipitate method. The transfection mixture remained on the cells for $7 \mathrm{~h}$ before the transfection medium was replaced with fresh medium. The supernatant from vector-producing $293 \mathrm{~T}$ cells was recovered every $22 \mathrm{~h}$ during 3 days before being harvested, passed through a $0.45-\mu \mathrm{m}$-pore-size filter to remove producer cells, and then subjected to two centrifugations at $4^{\circ} \mathrm{C}$ and $22,000 \times g$ for 90 minutes to concentrate the virus. The virus-containing pellet was dissolved in $1 \%$ bovine serum albumin. Viral concentrate $(20 \mu \mathrm{l})$ from pRV-Gsx2-IRES-EGFP or pRV-IRES-EGFP was used to transduce $3.5 \times 10^{6}$ dissociated NPCs in a 6-well plate as described previously [51]. Transduced NPCs were growth as neurospheres as described above for 5 DIV before being pelleted for RNA extraction.

\section{Generation of the anti-Nolz1 antibody}

Anti-Nolz1 polyclonal antibodies were obtained from the serum of immunized rabbits with a keyhole limpet hemocyanin (KLH)-conjugated oligopeptide coding for amino acids 2 to 14 of the Nolz1 sequence (MSTAPSLSALRSSKH; Figure 1A). Pre-immune serum was obtained from the same rabbits before immunization.

\section{Immunolabeling}

All immunostaining was performed using the following antibodies: polyclonal anti-NolzI $(1: 10,000)$, monoclonal anti-GFAP (1:500; Sigma Chemical Co.), monoclonal anti-Tuj1 (1:500; Sigma Chemical Co.), monoclonal antiBrdU (1:50; Dako A/S, Glostrup, Denmark), monoclonal anti-MAP2 (1:200; Sternberger Monoclonals, Lutherville, MD, USA), polyclonal anti-nestin (Rat 401; 1:40; Developmental Studies Hybridoma Bank; The University of Iowa, Iowa), polyclonal anti-Tle4 (1:200; generous gift from Dr Stefano Stifani, McGill University), polyclonal conjugated FITC-GFP (1:200; ABCAM, Cambridge, UK), polyclonal anti-Ki67 (1:200; Thermo Fisher Scientific SLU, Alcobendas, Madrid, Spain). For Nolz negative controls, pre-immunization serum was used at the same 
concentration as NolzI antibody-containing serum. Preparations were counterstained with DAPI to visualize the nucleus.

No signal was detected in control immunostaining assays in which the primary antibody was omitted.

\section{In situ hybridization}

We analyzed the expression of several genes by radioactive in situ hybridization as described elsewhere [52]. The following oligonucleotide probes were used: mouse NolzI - complementary to nucleotides 3,226 to 3,266 of the NolzI sequence (GenBank accession number NM_145459); mouse Tle1 - complementary to nucleotides 1,942 to 1,983 of the Tle 1 sequence (GenBank accession number NM_011599); mouse Tle2 - complementary to nucleotides 1,351 to 1,389 of the Tle 2 sequence (GenBank accession number NM_019725); mouse Tle3 - complementary to nucleotides 3,434 to 3,474 of the Tle3 sequence (GenBank accession number NM_001083927); mouse Tle4 - complementary to nucleotides 1,614 to 1,652 of the Tle4 sequence (GenBank accession number NM_0011600).

\section{Quantitative PCR assays}

Expression of several genes was evaluated by Q-PCR assays performed as previously described [15], using the following TaqMan ${ }^{\circledR}$ gene expression assays (Applied Biosystems): 18S, Hs99999901_s1; nestin, Mm00450205_m1; $\beta$-tubulin III, Mm00727586_s1; GFAP, Mm00546086_m1; MAP2, Mm00485230_m1; Gsx2, Mm00446650_m1; mouse NolzI, Mm00520908_m1; Raldh3, Mm0047 4049_m1; RAR $\alpha, M m 00436264$ m1; RAR $\beta, M m 0131$ 9674_m1; RAR $\gamma$, Mm00441083_m1; CRBP1, Mm00 441119_m1; Cyp26b1, Mm00558507_m1; Tle4, Mm011 95160_m1. To specifically recognize $h N o l z$, a customized Taqman $^{\oplus}$ Assay was designed consisting of the following primers: forward, CCTCGCCCTCCTCCAAAC; reverse, GCCCGATTTGGTGTCCTTGT; reporter, TCTCCTCG GTTGCCTCC. To provide negative controls and exclude contamination by genomic DNA, the reverse transcriptase was omitted in the cDNA synthesis step, and the samples were subjected to the PCR reaction with each TaqMan ${ }^{\circledR}$ gene expression assay.

Analysis and quantification was performed with the Comparative Quantitation Analysis program of the $\mathrm{MxPro}^{\mathrm{TM}} \mathrm{Q}-\mathrm{PCR}$ analysis software version 3.0 (Stratagene, La Jolla, CA, USA), using the $18 \mathrm{~S}$ gene expression as internal loading control. All Q-PCR assays were performed in duplicate and repeated for at least three independent experiments. The results were expressed as relative levels with respect to the expression of the same gene in the control condition, considered as $100 \%$.

\section{Western blotting}

We analyzed the levels of Nolz1 protein in transfected neurospheres or in the striatum at different developmental stages. Samples (at least $n=3$ per time point) were prepared and processed for western blotting as described elsewhere [53]. Blots were incubated overnight at $4^{\circ} \mathrm{C}$ with anti-NolzI antibody $(1: 50,000)$. The secondary antibody was a horse radish peroxidase-conjugated anti-rabbit IgG (1:3,000; Promega Biotech Iberica, SL., Madrid, Spain) and the signal was developed using the ECL western blotting analysis system (GE Healthcare Europe GMBH, Cerdanyola del Vallès, Barcelona, Spain).

\section{Cell counts}

In order to determine the role of NolzI on the proliferation of progenitor cells in vitro, we counted the number of cells that incorporate BrdU. BrdU- and EGFP-positive (transfected) cells were detected by immunocytochemistry and the total number of cells determined by DAPI counterstaining. For over-expressing experiments the results were expressed as the percentage of proliferating cells with respect to the transfected (EGFP-positive) cells, while for siRNA experiments, the results were expressed as the percentage of proliferating cells with respect the total number of cells $(n=5)$. We also counted by phase contrast the total number of neurospheres 5 days after $h N o l z$ or control transfection.

Cell death was evaluated by counting the number of apoptotic nuclei stained with DAPI after hNolz overexpression. The results were expressed as the percentage of dying cells with respect to transfected (EGFP-positive) cells $(n=4)$. Results were normalized with respect to control-transfected NSCs (considered as 100\%).

We estimated the cell cycle time as previously described $[1,29]$. Briefly, BrdU was added to neurosphere cultures during the last 1, 3, 6, 12 and 24 h of culturing. The number of BrdU-positive cells and the total number of cells, determined by DAPI counterstained nuclei, in each neurosphere was counted. The percentage of proliferating cells was calculated for each time point after BrdU administration. Regression analyses of the active portion of each BrdU labeling curve were used to estimate cell cycle time assuming that all cells proliferate at the same rate and that every cell is labeled at the end of a single cycle [29]. The $r^{2}$ of this lineal correlation was used to calculate the cell cycle duration. We counted at least 30 neurospheres in each condition in 3 transfected cultures.

We analyzed the cell cycle index as the number of cells that retain BrdU but leave the cell cycle (Ki67negative cells) after a 3-DIV pulse label. Thus, we 
counted the fraction of BrdU+/Ki67- cells and normalized to the total number of BrdU-positive cells in the culture. Results were expressed as absolute percentages in each condition.

To determine the effect of Nolz1 on the differentiation of LGE primary cultures, we counted the number of cells per coverslip overexpressing hNolz or EGFP that colocalized with different markers, such as nestin, Tuj1 and MAP2 3 or 5 days after the transfection. The results are expressed as the percentage of transfected cells colocalizing with the different markers with respect to the total number of transfected cells. Between 50 and 200 transfected cells per coverslip were counted per transfection ( $\mathrm{n}=3$ to 5$)$.

\section{Brain slice electroporation}

Coronal brain slices $(250 \mu \mathrm{m})$ from E14.5 mice embryos were obtained with a vibratome. Slices were plated onto culture membranes with minimum essential medium supplemented with $10 \% \mathrm{FBS}$ and $50 \mathrm{U} / \mathrm{ml}$ penicillinstreptomycin. After 1 hour in the incubator, media was changed to Neurobasal supplemented with B27, $1 \mathrm{mM}$ HEPES, $50 \mathrm{U} / \mathrm{ml}$ penicillin-streptomycin and $2 \mathrm{mM} \mathrm{L}$ glutamine. Two hours later, slices were electroporated with $8 \mu \mathrm{g}$ of DsRED-Nolz or DsRED plasmid. After 48 hours in culture, electroporated slices were fixed during 2 hours with 4\% PFA, dehydrated with increasing ethanol concentrations and stored until processing for immunohistochemistry.

We counted the number of Ki67-positive cells present in the electroporated zone of brain slices. First, we took a picture of the slices showing the RED fluorescence of the electroporated plasmids prior to fixation. Then, the slices were immunostained against Ki67 and the number of positive cells included in the electroporated area for the control side (DsRED plasmid) or experimental side (Nolz1-DsRED) were counted. The area to count was fixed by delineating the electroporated DsRED positive area in the pre-immunostaining image using ImageJ, and transferring this area to the Ki67 stained image. At least four different slices were counted for each condition.

\section{Luciferase RARE reporter assay}

To monitor the RARE activity we used the Cignal RARE Reporter Assay Kit (SABioscience Corporation, Frederick, MD, USA) following the manufacturer's instructions. Mixed neuron-glial LGE primary cultures were performed as described above and 24 hours after seeding cells were transfected with the inducible RAREresponsive mixture and the pLV-Nolz-IRES-EGFP or the pLV-IRES-EGFP plasmids. The transfection was performed using Lipofectamine LTX (Invitrogen SA) following the manufacturer's instructions. Two days later, cultures were processed to evaluate luciferase using the Dual-Luciferase Reporter Assay System (Promega). The firefly/Renilla luciferase ratio was calculated for each well, and results are expressed as the mean of four independent experiments and normalized with respect to control-transfected primary cultures (considered as $100 \%)$.

\section{Statistical analyses}

All results are expressed as the mean of independent experiments \pm standard error of the mean. Results were analyzed using the Student's $t$-test or one-way ANOVA followed by the Bonferroni post-hoc test.

\section{Abbreviations}

BrdU: bromodeoxyuridine; DEAB: 4-diethylaminobenzaldehyde; DIV: days in vitro; dLGE: dorsal LGE; DMSO: dimethyl sulfoxide; E: embryonic day; EGFP: enhanced green fluorescent protein; elB: elbow B; FBS: fetal bovine serum; GFAP: glial fibrillary acidic protein; GFP: green fluorescent protein; Gro: Groucho; hNolz: human Nolz; LGE: lateral ganglionic eminence; MAP: microtubule-associated protein; MCS: multiple cloning site; MZ: mantle zone; NET: NocA-Elbow (elB)-TIp; NPC: neural progenitor cell; PFA: paraformaldehyde; Q-PCR: quantitative PCR; RA: retinoic acid; Raldh: retinaldhehyde dehydrogenase; RAR: RA receptor; RARE: RA response element; siRNA: small interfering RNA; SVZ: subventricular zone; TLE: NocAelB-TIp; Tuj1: $\beta$-III tubulin; vLGE: ventral LGE; VZ: ventricular zone.

\section{Acknowledgements}

We would like to thank M Teresa Muñoz, Ana López and Josep Barrachina for technical assistance, and Dr Maria Calvo, Anna Bosch and Neus Abella from the confocal microscopy unit at the Serveis Científico-Tècnics (Universitat de Barcelona) for their support and advice on confocal techniques. We also thank Dr Peter Marynen (Université de Leuven, Belgium) for the pcDNA-hGsx2 construct and Dr Pantelis Tsoulfas (University of Miami, FL, USA) for the pRRLsinPPT plasmid. We thank Dr Stefano Stifani (McGill University, Montreal, Canada) for the anti-Tle4 antibody. We are also grateful to Dr A Nagy, R Nagy, Dr J Rossant, and Dr W Abramow-Newerly from the Samuel Lunenfeld Research Institute (Mont Sinai Hospital, Toronto, Canada) for the generous gift of the R1 mouse embryonic stem cell line. This study was supported by grants from the Ministerio de Ciencia e Innovación (SAF2008-04360, to JA; SAF2009-07774 and PLE2009-0089, to JMC; SAF200405798, to CV-A), Spain; Instituto de Salud Carlos III, Ministerio de Ciencia e Innovación (CIBERNED, to JA and CV-A; and RETICS (RD06/0010/0006; Red de Terapia Celular), to JMC), Spain; the Fundació La Marató de TV3 (to JA), Spain; Generalitat de Catalunya (2009SGR-00326 to JA), Spain; and the National Institutes of Health (EY013969, to GD). The Cell Therapy Program is supported by the Centre of Regenerative Medicine in Barcelona (CMRB; Promt-0901, to JMC; Generalitat de Catalunya), Spain.

\section{Author details}

${ }^{1}$ Department of Cell Biology, Immunology and Neuroscience, Faculty of Medicine, IDIBAPS, Universitat de Barcelona, C/Casanova 143, 08036 Barcelona, Spain. ${ }^{2}$ Centro de Investigación Biomédica en Red sobre Enfermedades Neurodegenerativas (CIBERNED), Spain. ${ }^{3}$ Department of Molecular Neurobiology, National Institute for Medical Research, The Ridgeway, Mill Hill, London NW7 1AA, UK. ${ }^{4}$ Cell Therapy Program, Faculty of Medicine, Universitat de Barcelona, C/Casanova 143, 08036 Barcelona, Spain ${ }^{5}$ Alicante Neuroscience Institute, Miguel Hernandez University, Consejo Superior de Investigaciones Científicas (CSIC), 03550 San Juan de Alicante, Spain. ${ }^{6}$ Departamento de Neurobiología Molecular, Celular y del Desarrollo, Instituto Cajal, Consejo Superior de Investigaciones Científicas (CSIC), C/ Doctor Arce 37, 28002 Madrid, Spain. Division of Developmental Biology, Children's Hospital Research Foundation, 3333 Burnet Avenue, Cincinnati, OH 45229, USA. ${ }^{8}$ Development and Aging Program, Sanford-Burnham Medical Research Institution, 10901 North Torrey Pines Road, La Jolla, CA 92093, USA. 
9Departamento de Química Orgánica, Universidade de Vigo, LagoasMarcosende s/n, 36310 Vigo, Spain.

\section{Authors' contributions}

$\mathrm{NU}$, collection and assembly of data, data analysis and interpretation, manuscript writing, final approval of manuscript; RM-I, CH, ME, EC, MP, HRMG, RW, CC, SA, RA, collection and assembly of data, final approval of manuscript; GD, KC, ARdL, CV-A, SM, JA, financial support, data analysis and interpretation, final approval of manuscript; JMC, conception and design, financial support, administrative support, data analysis and interpretation, manuscript writing, final approval of manuscript.

\section{Competing interests}

The authors declare that they have no competing interests.

Received: 19 April 2010 Accepted: 24 August 2010

Published: 24 August 2010

\section{References}

1. Bhide PG: Cell cycle kinetics in the embryonic mouse corpus striatum. J Comp Neurol 1996, 374:506-522.

2. Takahashi T, Nowakowski RS, Caviness VS Jr: The cell cycle of the pseudostratified ventricular epithelium of the embryonic murine cerebral wall. J Neurosci 1995, 15:6046-6057.

3. Haubensak W, Attardo A, Denk W, Huttner WB: Neurons arise in the basal neuroepithelium of the early mammalian telencephalon: a major site of neurogenesis. Proc Natl Acad Sci USA 2004, 101:3196-3201.

4. Miyata T, Kawaguchi A, Saito K, Kawano M, Muto T, Ogawa M: Asymmetric production of surface-dividing and non-surface-dividing cortical progenitor cells. Development 2004, 131:3133-3145.

5. Noctor SC, Martinez-Cerdeno V, Ivic L, Kriegstein AR: Cortical neurons arise in symmetric and asymmetric division zones and migrate through specific phases. Nat Neurosci 2004, 7:136-144.

6. Haubst N, Berger J, Radjendirane V, Graw J, Favor J, Saunders GF, Stoykova A, Gotz M: Molecular dissection of Pax6 function: the specific roles of the paired domain and homeodomain in brain development. Development 2004, 131:6131-6140.

7. Marklund M, Sjodal M, Beehler BC, Jessell TM, Edlund T, Gunhaga L: Retinoic acid signalling specifies intermediate character in the developing telencephalon. Development 2004, 131:4323-4332.

8. Duester G: Retinoic acid synthesis and signaling during early organogenesis. Cell 2008, 134:921-931.

9. Li H, Wagner E, McCaffery P, Smith D, Andreadis A, Drager UC: A retinoic acid synthesizing enzyme in ventral retina and telencephalon of the embryonic mouse. Mech Dev 2000, 95:283-289.

10. Mic FA, Haselbeck RJ, Cuenca AE, Duester G: Novel retinoic acid generating activities in the neural tube and heart identified by conditional rescue of Raldh2 null mutant mice. Development 2002, 129:2271-2282.

11. Molotkova N, Molotkov A, Duester G: Role of retinoic acid during forebrain development begins late when Raldh3 generates retinoic acid in the ventral subventricular zone. Dev Biol 2007, 303:601-610.

12. Liao WL, Liu FC: RARbeta isoform-specific regulation of DARPP-32 gene expression: an ectopic expression study in the developing rat telencephalon. Eur J Neurosci 2005, 21:3262-3268.

13. Gerfen CR: The neostriatal mosaic: multiple levels of compartmental organization. Trends Neurosci 1992, 15:133-139.

14. Chatzi C, Scott RH, Pu J, Lang B, Nakamoto C, McCaig CD, Shen S: Derivation of homogeneous GABAergic neurons from mouse embryonic stem cells. Exp Neurol 2009, 217:407-416.

15. Martin-Ibanez R, Urban N, Sergent-Tanguy S, Pineda JR, Garrido-Clua N, Alberch J, Canals JM: Interplay of leukemia inhibitory factor and retinoic acid on neural differentiation of mouse embryonic stem cells. J Neurosci Res 2007, 85:2686-2701.

16. Corbin JG, Gaiano N, Machold RP, Langston A, Fishell G: The Gsh2 homeodomain gene controls multiple aspects of telencephalic development. Development 2000, 127:5007-5020.

17. Yun $K$, Potter S, Rubenstein JL: Gsh2 and Pax6 play complementary roles in dorsoventral patterning of the mammalian telencephalon. Development 2001, 128:193-205.
18. Waclaw RR, Wang B, Campbell K: The homeobox gene Gsh2 is required for retinoid production in the embryonic mouse telencephalon. Development 2004, 131:4013-4020.

19. Chang CW, Tsai CW, Wang HF, Tsai HC, Chen HY, Tsai TF, Takahashi H, Li HY, Fann MJ, Yang CW, Hayashizaki Y, Saito T, Liu FC: Identification of a developmentally regulated striatum-enriched zinc-finger gene, Nolz-1, in the mammalian brain. Proc Natl Acad Sci USA 2004, 101:2613-2618.

20. Ji SJ, Periz G, Sockanathan S: Nolz1 is induced by retinoid signals and controls motoneuron subtype identity through distinct repressor activities. Development 2009, 136:231-240.

21. Cheah PY, Meng YB, Yang X, Kimbrell D, Ashburner M, Chia W: The Drosophila I(2)35Ba/nocA gene encodes a putative $\mathrm{Zn}$ finger protein involved in the development of the embryonic brain and the adult ocellar structures. Mol Cell Biol 1994, 14:1487-1499.

22. Dorfman R, Glazer L, Weihe U, Wernet MF, Shilo BZ: Elbow and Noc define a family of zinc finger proteins controlling morphogenesis of specific tracheal branches. Development 2002, 129:3585-3596.

23. Hoyle J, Tang YP, Wiellette EL, Wardle FC, Sive H: nlz gene family is required for hindbrain patterning in the zebrafish. Dev Dyn 2004, 229:835-846.

24. Runko AP, Sagerstrom CG: Nlz belongs to a family of zinc-fingercontaining repressors and controls segmental gene expression in the zebrafish hindbrain. Dev Biol 2003, 262:254-267.

25. Runko AP, Sagerstrom CG: Isolation of nlz2 and characterization of essential domains in $\mathrm{Nlz}$ family proteins. J Biol Chem 2004, 279:11917-11925.

26. McGlinn E, Richman JM, Metzis V, Town L, Butterfield NC, Wainwright BJ, Wicking C: Expression of the NET family member Zfp503 is regulated by hedgehog and BMP signaling in the limb. Dev Dyn 2008, 237:1172-1182.

27. Von OT, Syu LJ, Mellerick DM: Conserved properties of the Drosophila homeodomain protein, Ind. Mech Dev 2007, 124:925-934.

28. Stenman J, Yu RT, Evans RM, Campbell K: Tlx and Pax6 co-operate genetically to establish the pallio-subpallial boundary in the embryonic mouse telencephalon. Development 2003, 130:1113-1122.

29. Kippin TE, Martens DJ, van der KD: p21 loss compromises the relative quiescence of forebrain stem cell proliferation leading to exhaustion of their proliferation capacity. Genes Dev 2005, 19:756-767.

30. Chenn A, Walsh CA: Regulation of cerebral cortical size by control of cell cycle exit in neural precursors. Science 2002, 297:365-369.

31. Verma AK, Shoemaker A, Simsiman R, Denning M, Zachman RD: Expression of retinoic acid nuclear receptors and tissue transglutaminase is altered in various tissues of rats fed a vitamin A-deficient diet. J Nutr 1992, 122:2144-2152.

32. Wang B, Waclaw RR, Allen ZJ, Guillemot F, Campbell K: Ascl1 is a required downstream effector of Gsx gene function in the embryonic mouse telencephalon. Neural Dev 2009, 4:5.

33. Russo D, Regazzi M, Sacchi S, Visani G, Lazzarino M, Avvisati G, Pelicci PG, Dastoli G, Grandi C, lacona I, Candoni A, Grattoni R, Galieni P, Rupoli S, Liberati AM, Maiolo AT: All-trans retinoic acid (ATRA) in patients with chronic myeloid leukemia in the chronic phase. Leukemia 1998, 12:449-454.

34. Alvarez S, Alvarez R, Khanwalkar H, Germain P, Lemaire G, RodriguezBarrios F, Gronemeyer H, de Lera AR: Retinoid receptor subtype-selective modulators through synthetic modifications of RARgamma agonists. Bioorg Med Chem 2009, 17:4345-4359.

35. Alvarez S, Khanwalkar H, Alvarez R, Erb C, Martinez C, Rodriguez-Barrios F, Germain P, Gronemeyer H, de Lera AR: C3 halogen and c8" substituents on stilbene arotinoids modulate retinoic Acid receptor subtype function. Chem Med Chem 2009, 4:1630-1640.

36. Germain P, Kammerer S, Perez E, Peluso-lltis C, Tortolani D, Zusi FC, Starrett J, Lapointe P, Daris JP, Marinier A, de Lera AR, Rochel N, Gronemeyer H: Rational design of RAR-selective ligands revealed by RARbeta crystal stucture. EMBO Rep 2004, 5:877-882.

37. Zaki PA, Quinn JC, Price DJ: Mouse models of telencephalic development. Curr Opin Genet Dev 2003, 13:423-437.

38. Toresson H, Parmar M, Campbell K: Expression of Meis and Pbx genes and their protein products in the developing telencephalon: implications for regional differentiation. Mech Dev 2000, 94:183-187.

39. Edlund T, Jessell TM: Progression from extrinsic to intrinsic signaling in cell fate specification: a view from the nervous system. Cell 1999, 96:211-224. 
40. McCaffery P, Drager UC: High levels of a retinoic acid-generating dehydrogenase in the meso-telencephalic dopamine system. Proc Natl Acad Sci USA 1994, 91:7772-7776.

41. Campbell K: Dorsal-ventral patterning in the mammalian telencephalon. Curr Opin Neurobiol 2003, 13:50-56.

42. Waclaw RR, Wang B, Pei Z, Ehrman LA, Campbell K: Distinct temporal requirements for the homeobox gene Gsx2 in specifying striatal and olfactory bulb neuronal fates. Neuron 2009, 63:451-465.

43. Toresson H, Mata dU, Fagerstrom C, Perlmann T, Campbell K: Retinoids are produced by glia in the lateral ganglionic eminence and regulate striatal neuron differentiation. Development 1999, 126:1317-1326.

44. Liao WL, Tsai HC, Wang HF, Chang J, Lu KM, Wu HL, Lee YC, Tsai TF, Takahashi H, Wagner M, Ghyselinck NB, Chambon P, Liu FC: Modular patterning of structure and function of the striatum by retinoid receptor signaling. Proc Natl Acad Sci USA 2008, 105:6765-6770.

45. Zetterstrom RH, Lindqvist E, Mata dU, Tomac A, Eriksson U, Perlmann T, Olson L: Role of retinoids in the CNS: differential expression of retinoid binding proteins and receptors and evidence for presence of retinoic acid. Eur J Neurosci 1999, 11:407-416.

46. Rajaii F, Bitzer ZT, Xu Q, Sockanathan S: Expression of the dominant negative retinoid receptor, RAR403, alters telencephalic progenitor proliferation, survival, and cell fate specification. Dev Biol 2008, 316:371-382.

47. Dehni G, Liu Y, Husain J, Stifani S: TLE expression correlates with mouse embryonic segmentation, neurogenesis, and epithelial determination. Mech Dev 1995, 53:369-381.

48. Koop KE, MacDonald LM, Lobe CG: Transcripts of Grg4, a murine groucho-related gene, are detected in adjacent tissues to other murine neurogenic gene homologues during embryonic development. Mech Dev 1996, 59:73-87.

49. Yao J, Liu Y, Husain J, Lo R, Palaparti A, Henderson J, Stifani S: Combinatorial expression patterns of individual TLE proteins during cell determination and differentiation suggest non-redundant functions for mammalian homologs of Drosophila Groucho. Dev Growth Differ 1998, 40:133-146.

50. Naldini L, Blomer U, Gage FH, Trono D, Verma IM: Efficient transfer, integration, and sustained long-term expression of the transgene in adult rat brains injected with a lentiviral vector. Proc Natl Acad Sci USA 1996, 93:11382-11388.

51. Otaegi G, Yusta-Boyo MJ, Vergano-Vera E, Mendez-Gomez HR, Carrera AC, Abad JL, Gonzalez M, de la Rosa EJ, Vicario-Abejon C, de Pablo F: Modulation of the PI 3-kinase-Akt signalling pathway by IGF-I and PTEN regulates the differentiation of neural stem/precursor cells. J Cell Sci 2006, 119:2739-2748

52. Marco S, Canudas AM, Canals JM, Gavalda N, Perez-Navarro E, Alberch J: Excitatory amino acids differentially regulate the expression of GDNF, neurturin, and their receptors in the adult rat striatum. Exp Neurol 2002, 174:243-252.

53. Canals JM, Pineda JR, Torres-Peraza JF, Bosch M, Martin-lbanez $R$, Munoz MT, Mengod G, Ernfors P, Alberch J: Brain-derived neurotrophic factor regulates the onset and severity of motor dysfunction associated with enkephalinergic neuronal degeneration in Huntington's disease. $J$ Neurosci 2004, 24:7727-7739.

\section{doi:10.1186/1749-8104-5-21}

Cite this article as: Urbán et al:: Nolz1 promotes striatal neurogenesis through the regulation of retinoic acid signaling. Neural Development 2010 5:21.

\section{Submit your next manuscript to BioMed Central and take full advantage of:}

- Convenient online submission

- Thorough peer review

- No space constraints or color figure charges

- Immediate publication on acceptance

- Inclusion in PubMed, CAS, Scopus and Google Scholar

- Research which is freely available for redistribution 